\title{
Size- and temperature-dependent magnetization of iron nanoclusters
}

\author{
G. Dos Santos $\odot,{ }^{1,2, *}$ R. Aparicio, ${ }^{1,2}$ D. Linares, ${ }^{3}$ E. N. Miranda, ${ }^{4}$ J. Tranchida, ${ }^{5}$ G. M. Pastor, ${ }^{6}$ and E. M. Bringa $\oplus^{1,2,7}$ \\ ${ }^{1}$ CONICET, Mendoza 5500, Argentina \\ ${ }^{2}$ Facultad de Ingeniería, Universidad de Mendoza, Mendoza 5500, Argentina \\ ${ }^{3}$ Departamento de Física, Instituto de Física Aplicada, Universidad Nacional de San Luis-CONICET, Ejército de Los Andes 950, \\ D5700HHW San Luis, Argentina \\ ${ }^{4}$ IANIGLA-CONICET, CCT Mendoza, 5500-Mendoza, Argentina \\ ${ }^{5}$ Computational Multiscale, Center for Computing Research, Sandia National Laboratories, P.O. Box 5800, MS 1322, Albuquerque, New \\ Mexico 87185, USA \\ ${ }^{6}$ Institute of Theoretical Physics, University of Kassel, 34134 Kassel, Germany \\ ${ }^{7}$ Centro de Nanotecnología Aplicada, Facultad de Ciencias, Universidad Mayor, Santiago 8580745, Chile
}

(Received 25 August 2020; revised 30 October 2020; accepted 2 November 2020; published xxxxxxxxx)

\begin{abstract}
The magnetic behavior of bcc iron nanoclusters, with diameters between 2 and $8 \mathrm{~nm}$, is investigated by means of spin dynamics simulations coupled to molecular dynamics, using a distance-dependent exchange interaction. Finite-size effects in the total magnetization as well as the influence of the free surface and the surface/core proportion of the nanoclusters are analyzed in detail for a wide temperature range, going beyond the cluster and bulk Curie temperatures. Comparison is made with experimental data and with theoretical models based on the mean-field Ising model adapted to small clusters, and taking into account the influence of low coordinated spins at free surfaces. Our results for the temperature dependence of the average magnetization per atom $M(T)$, including the thermalization of the transnational lattice degrees of freedom, are in very good agreement with available experimental measurements on small Fe nanoclusters. In contrast, significant discrepancies with experiment are observed if the translational degrees of freedom are artificially frozen. The finite-size effects on $M(T)$ are found to be particularly important near the cluster Curie temperature. Simulated magnetization above the Curie temperature scales with cluster size as predicted by models assuming short-range magnetic ordering. Analytical approximations to the magnetization as a function of temperature and size are proposed.
\end{abstract}

DOI: 10.1103/PhysRevB.00.004400

\section{INTRODUCTION}

At the nanoscale, finite-size effects can strongly influence the magnetic properties of materials [1]. Fe layers deposited on $\mathrm{W}$ substrates (typically on the order of few hundreds of Fe atoms) are a prototypical example of those effects [2]. Numerical and experimental studies have extensively demonstrated the impact of the size, dimension, and number of Fe monolayers on their magnetic properties, including ordering temperature (Curie or Néel), magnetic susceptibility, or magnon dispersion relations [3-5]. In the field of magnetic nanoclusters, large departures from the corresponding bulk magnetic properties have also been observed. For example, hysteresis loop, coercive field, ordering temperature, or spontaneous magnetization, have been shown to drastically depend on the size of iron oxide nanoparticles (NPs) [6-8].

Understanding magnetism at the nanoscale is important since the computed or measured magnetic properties can be used to parametrize micromagnetic models, which are extremely valuable to simulate technological applications [9]. This is the case, for example, even in the most basic StonerWohlfarth (SW) model [10], which represents the coercivity

*gonzalodossantos@gmail.com and switching field of a magnetic single-domain NP. In its simplest form, the SW model depends on the total magnetization and anisotropy energy of the particle. It is therefore of fundamental importance to develop accurate numerical tools evaluating how the cluster magnetization is affected by temperature and particle size. Electronic first-principles calculations are certainly extremely valuable to obtain better insight at localized effects and to derive magnetic interaction parameters [11]. However, the involved computational costs and their poor scalability makes them unpractical for simulating NPs in the size range of technological interest, as they are typically limited to systems up to a few hundreds of atoms $[12,13]$. Consequentely, developing reliable novel approaches is crucial for the progress in this field.

Leveraging an adiabatic atomistic spin approximation [14], atomistic spin dynamics (ASD) is a widely used classical spinlattice methodology allowing us to model complex nanoscale systems [15-18]. Magnetic trajectories are simulated on a potential energy surface generated by a magnetic Hamiltonian usually parametrized from first-principles calculations [19]. Assuming fixed atomic positions, ASD simulations do not account for magnon-phonon interactions. Former studies have displayed the importance of those interactions on the description of materials properties such as magnon lifetime, phononic thermal conductivity, magnetic switching or critical 
temperature [20-22]. Spin dynamics (SD) can also be used as a coarse grained approach, to compute the time evolution of blocks of the systems having many atoms with some effective magnetization. This allows the simulation of micron-sized systems that are of technological relevance [23-26]. ASD has been recently applied to $\mathrm{Fe}$ oxide NPs, using a triangular lattice [27].

A study accounting for the magnon-phonon interactions in molecular dynamics (MD) simulations has been performed by Dudarev and Derlet. Using a combination of the Stoner and the Ginzburg-Landau models, they developed a "magnetic" potential for $\alpha$ iron to take into account some effects of magnetism, including the energetics of point defects [28,29]. Later, a numerical methodology coupling MD and ASD by explicitly treating atomic and spin degrees of freedom as well as their coupling through a magnetoelastic Hamiltonian was presented by Ma et al. [30]. Recently, a large number of investigations have been carried out applying MD coupled to spin dynamics simulations (MD-SD) to explain experiments of magnetic instability [31], demagnetization, impact of temperature on magnetomechanical properties, and phase transitions [21,32]. This includes the development of a new software for the implementation of the model, SPILADY [33]. Perera et al. carried out studies of magnetic Fe using MD-SD [34], including spin-orbit coupling effects [35]. Other recent studies incorporate additional exchange parameters obtained from ab initio methods [36].

In this paper, SD coupled to classical MD simulations [37] is used to incorporate thermal spin and mechanical effects which are difficult to include in ASD simulations. This method is applied to Fe nanoclusters to obtain magnetization versus size and temperature, subsequently comparing those results to semianalytical models.

The paper is organized as follows. In Sec. II, the simulation framework employed is presented, as well as the details of the calculations. In Sec. III, two semianalytical models are introduced to qualitatively analyze numerical simulations. The results are presented and discussed in Sec. IV. Finally, the main conclusions are drawn in Sec. V.

\section{METHODS}

\section{A. Theoretical framework}

In this paper, we perform MD-SD, where the spin degrees of freedom are coupled to the lattice degrees of freedom. For this purpose, we run our simulations under the SPIN package recently added to the software LAMMPS [37]. Under this framework, one is able to introduce magnetic effects in a classical MD simulation through a generalized Hamiltonian:

$$
\mathcal{H}=\sum_{i=1}^{N} \frac{\left|\boldsymbol{p}_{i}\right|^{2}}{2 m_{i}}+\sum_{i, j, i \neq j}^{N} V\left(r_{i j}\right)+\mathcal{H}_{\text {mag }} .
$$

The first term is the kinetic energy of the atoms and the second term is a classical interatomic potential describing the mechanical interactions between the atoms. The last term is a magnetic Hamiltonian, which can contain several terms, accounting for spin-spin exchange interactions, magnetic anisotropy (cubic, uniaxial), Zeeman, dipolar, DzyaloshinskiiMoriya, and magnetoelectric interactions. In this paper, we will consider isolated Fe NPs, with no interaction with external magnetic fields. The simplified magnetic Hamiltonian used in the present work is given by

$$
\mathcal{H}_{\text {mag }}=-\sum_{i, j, i \neq j}^{N} J\left(r_{i j}\right) \boldsymbol{s}_{i} \cdot \boldsymbol{s}_{j}+\mathcal{H}_{\text {cubic }} .
$$

The first term is a Heisenberg Hamiltonian accounting for spin-spin interactions, where $\boldsymbol{s}_{i}$ is the normalized spin vector of spin $i$ and $J\left(r_{i j}\right)$ is the Heisenberg magnetic coupling exchange constant, which depends on the distance $r_{i j}$ between atoms $i$ and $j$. The second term accounts for cubic magnetic anisotropy and is given by

$$
\begin{aligned}
\mathcal{H}_{\text {cubic }}= & \sum_{i=1}^{N} K_{1}\left[\left(\boldsymbol{s}_{i} \cdot \boldsymbol{n}_{1}\right)^{2}\left(\boldsymbol{s}_{i} \cdot \boldsymbol{n}_{2}\right)^{2}+\left(\boldsymbol{s}_{i} \cdot \boldsymbol{n}_{2}\right)^{2}\left(\boldsymbol{s}_{i} \cdot \boldsymbol{n}_{3}\right)^{2}\right. \\
& \left.+\left(\boldsymbol{s}_{i} \cdot \boldsymbol{n}_{1}\right)^{2}\left(\boldsymbol{s}_{i} \cdot \boldsymbol{n}_{3}\right)^{2}\right]+K_{2}\left(\boldsymbol{s}_{i} \cdot \boldsymbol{n}_{1}\right)^{2}\left(\boldsymbol{s}_{i} \cdot \boldsymbol{n}_{3}\right)^{2}\left(\boldsymbol{s}_{i} \cdot \boldsymbol{n}_{3}\right)^{2},
\end{aligned}
$$

where $K_{1}$ and $K_{2}$ are the intensity coefficients and $\boldsymbol{n}_{1}, \boldsymbol{n}_{2}$, and $\boldsymbol{n}_{3}$ are unitary vectors along the three anisotropic directions of the material. For bcc iron $\boldsymbol{n}_{1}=(100), \boldsymbol{n}_{2}=(010)$, and $\boldsymbol{n}_{3}=(001)$. The anisotropy term given by Eq. (3) applies a precession torque on each magnetic spin.

The central aspect of this simulation scheme is the addition of a classical spin vector $s_{i}$ to each atom $i$. This enables magnetic degrees of freedom to be explicitly treated and added to the atomic degrees of freedom, momentum $\boldsymbol{p}_{i}$, and position $\boldsymbol{r}_{i}$. The equations of motion (EOM) can be derived from the Hamiltonian of Eq. (1):

$$
\begin{gathered}
\frac{d \boldsymbol{r}_{i}}{d t}=\frac{\boldsymbol{p}_{i}}{m_{i}}, \\
\frac{d \boldsymbol{p}_{i}}{d t}=\sum_{i, j, i \neq j}^{N}\left[\frac{d V\left(r_{i j}\right)}{d r_{i j}}+\frac{d J\left(r_{i j}\right)}{d r_{i j}} \boldsymbol{s}_{i} \cdot \boldsymbol{s}_{j}\right] \boldsymbol{e}_{i}, \\
\frac{d \boldsymbol{s}_{i}}{d t}=\boldsymbol{w}_{i} \times \boldsymbol{s}_{i},
\end{gathered}
$$

where $\boldsymbol{e}_{i}$ is a unit vector along the direction of the vector $\boldsymbol{r}_{i j}$ and $\boldsymbol{w}_{i}$ is the analog of a spin force applied to spin $i$, defined as

$$
\boldsymbol{w}_{i}=-\frac{1}{\hbar} \frac{\partial \mathcal{H}_{\mathrm{mag}}}{\partial \boldsymbol{s}_{i}} .
$$

The distance dependence of the exchange constant $J\left(r_{i j}\right)$ on the first term of Eq. (2) is a key aspect of the model since it mediates the spin and lattice coupling. Furthermore, $J\left(r_{i j}\right)$ is modeled by a function based on the Bethe-Slater curve $[38,39]$, parameterized using three coefficients that must be fitted to $a b$ initio calculations:

$$
J\left(r_{i j}\right)=4 \alpha\left(\frac{r_{i j}}{\delta}\right)^{2}\left[1-\gamma\left(\frac{r_{i j}}{\delta}\right)^{2}\right] e^{-\left(\frac{r_{i j}}{\delta}\right)^{2}} \Theta\left(R_{c}-r_{i j}\right),
$$

where $\Theta\left(R_{c}-r_{i j}\right)$ is the Heaviside step function and $R_{c}$ is the cutoff distance. In the present paper, we have used the parametrization for bcc iron described in previous works $[20,37]$ from $a b$ initio calculations reported by Pajda et al. [40]. Specifically, the values of the fitting parameters are $\alpha=25.498 \mathrm{meV}, \gamma=0.281, \delta=0.1999 \mathrm{~nm}$, and exchange interaction cutoff distance $R_{c}=0.34 \mathrm{~nm}$. The same $J\left(r_{i j}\right)$ applies to all atoms in the NP. Notice that there is no general 
agreement about the exact value of the coupling-exchange constant for different interatomic distances for bcc Fe. In fact, the reported values of $J\left(r_{i j}\right)$ from $a b$ initio calculations found in the literature show large discrepancies [40-42].

Temperature in other simulation schemes, such as micromagnetic simulations, is not uniquely defined and has to be rescaled to compare with experiments [24]. In the simulations presented here, lattice and magnetic temperatures have a clear definition. The equilibrium lattice temperature is related to the kinetic temperature of the atoms and is defined as in classical MD simulations,

$$
T_{L}=\frac{2}{3 N k_{B} T} \sum_{i=1}^{N} \frac{\left|\boldsymbol{p}_{i}\right|^{2}}{2 m_{i}},
$$

where $k_{B}$ is the Boltzmann constant. Following the approach of Nurdin and Schotte [43], the temperature of the spin system is defined as follows:

$$
T_{S}=\frac{\hbar}{2 k_{B} T} \frac{\sum_{i=1}^{N}\left|\boldsymbol{s}_{i} \times \boldsymbol{w}_{i}\right|^{2}}{\sum_{i=1}^{N} \boldsymbol{s}_{i} \cdot \boldsymbol{w}_{i}} .
$$

In addition, in these spin-lattice simulations, the effect of temperature in lattice expansion is more realistic (due to the spin-lattice coupling) than in some previous approaches, which consider, for example, an homogeneous linear thermal expansion of the lattice with fixed atoms [44]. To thermalize the system and maintain a constant temperature, both the lattice and spin subsystems can be connected (separately) to a thermal bath. This connection is performed following the Langevin approach $[14,45]$. For the SD, this connection introduces a random torque and Eq. (6) is replaced by a stochastic Landau-Lifshitz-Gilbert equation, while for the lattice subsystem a damping term and a random force are added to Eq. (5). This yields the following EOM:

$$
\begin{gathered}
\frac{d \boldsymbol{r}_{i}}{d t}=\frac{\boldsymbol{p}_{i}}{m_{i}}, \\
\frac{d \boldsymbol{p}_{i}}{d t}=\sum_{i, j, i \neq j}^{N}\left[\frac{d V\left(r_{i j}\right)}{d r_{i j}}+\frac{d J\left(r_{i j}\right)}{d r_{i j}} \boldsymbol{s}_{i} \cdot \boldsymbol{s}_{j}\right] \boldsymbol{e}_{i} \\
-\frac{\gamma_{L}}{m_{i}} \boldsymbol{p}_{i}+\boldsymbol{f}(t), \\
\frac{d \boldsymbol{s}_{i}}{d t}=\frac{1}{1+\lambda^{2}}\left[\left(\boldsymbol{w}_{i}+\boldsymbol{\eta}(t)\right) \times \boldsymbol{s}_{i}+\lambda \boldsymbol{s}_{i} \times\left(\boldsymbol{w}_{i} \times \boldsymbol{s}_{i}\right)\right],
\end{gathered}
$$

where $\lambda$ and $\lambda_{L}$ are damping parameters for the spin and lattice subsystems, respectively. In addition, the random vector $\boldsymbol{\eta}(t)$ and the random fluctuating force $\boldsymbol{f}(t)$ are drawn from a Gaussian probability law with $\langle\boldsymbol{\eta}(t)\rangle=\langle\boldsymbol{f}(t)\rangle=0$. For extra details on thermostats and the properties of $\boldsymbol{\eta}(t)$ and $\boldsymbol{f}(t)$, see Ref. [37]. The interaction between atoms is modeled using an embedded atom model (EAM) interatomic potential [46] which describes reasonably well several Fe properties, including the thermal expansion, phonon dispersion curves, mean-square displacements, and surface relaxations. The interatomic cutoff distance for this potential was set to $0.35 \mathrm{~nm}$.

The simulations are performed using classical atomic dynamics and classical SD, without considering any quantummechanical effects. This is in line with the adiabatic approximation. However, the behavior at cryogenic temperatures

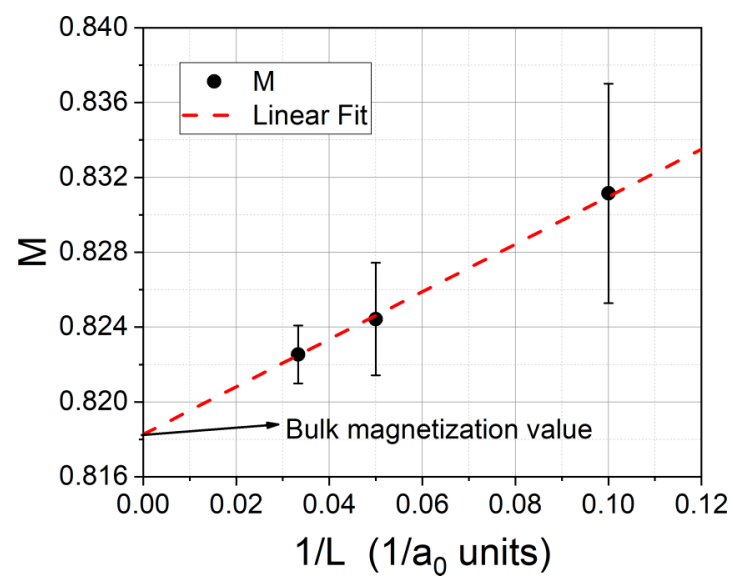

FIG. 1. Finite-size scaling for bulk magnetization simulations, using periodic boundary conditions. The data correspond to simulations run at $300 \mathrm{~K}$ and error bars show standard deviation resulting from averaging magnetization over the final $0.5 \mathrm{~ns}$.

might not be well described. Moreover, other effects resulting from electron-phonon and electron-spin coupling are not included. There are studies which include electrons within a "classical" two-temperature model approach for electrons and atoms, leading to a three-temperature model when spins are also included [33].

The SD is calculated using a Landau-Lifshitz-Gilbert approach [14], integrated with a Suzuki-Trotter integrator. Details can be found in Tranchida et al. [37].

\section{B. Simulation details}

Body centered cubic (bcc) iron NPs are simulated ranging their diameter and temperature from 2 to $8 \mathrm{~nm}$ and from 10 to $1300 \mathrm{~K}$, respectively. In addition, "bulk" simulations were run and used as a reference to address the finite-size effects of the NPs. In these cases, we have modeled cubic simulation boxes with linear sizes of $10 a_{0}, 20 a_{0}$, and $30 a_{0}$ (with $a_{0}=2.8665 \AA$ A the bcc Fe lattice parameter) containing 2000, 16000 , and 54000 atoms, respectively, and considering periodic boundary conditions in the three directions.

The magnetization values for the bulk systems were obtained from linear finite-size scaling analysis. As shown in Fig. 1, the magnetization values for the systems with $L=10$, 20 , and 30 are plotted as a function of $1 / L$, and then the bulk magnetization is determined as the intercept of a linear fit for those points.

All systems were simulated without external magnetic field, and considering cubic magnetic anisotropy along the three main axes of the bcc lattice (details of how the anisotropy is included in the simulations are given in Ref. [37]). The anisotropy constants were set to $K_{1}=$ $3.5 \mu \mathrm{eV} /$ atom and $K_{2}=0.36 \mu \mathrm{eV} /$ atom [47]. It has been argued that anisotropy values vary near the surface of NPs due to the reduction of coordination $[48,49]$. Nevertheless, as shown by Ellis and Chantrell [50], for example, the overall effect in FePt NPs of $4.632 \mathrm{~nm}$ and $2.316 \mathrm{~nm}$ is a change of about $10 \%$ in the anisotropy constant. In this paper, the anysotropy constant is assumed to be the same for all NPs, 


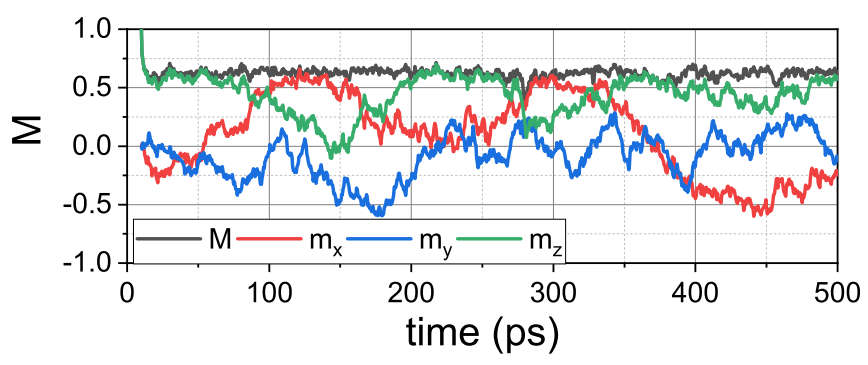

FIG. 2. Time dependence of the components $m_{x}, m_{y}, m_{z}$, of the magnetization along the $x, y$, and $z$ axes and the total magnetization $M$ during a typical simulation. The results correspond to simulations of a 2-nm-wide sphere at $400 \mathrm{~K}$.

despite surface proportion being clearly different for the sizes studied here.

Initially, all atomic spins were oriented along the $\mathrm{z}$ axis ([001] direction). This initial configuration was chosen since the magnetization reaches an equilibrium value faster than when the spins start from a random configuration (see Fig. $\mathrm{S} 1$ in the Supplemental Material [51]).

All simulations span more than $0.5 \mathrm{~ns}$, using a time step of 1.0 fs. All samples were initially thermalized to equilibrium using a Langevin thermostat applied to the lattice and another thermostat applied to the spins to ensure fast thermal equilibrium. In all cases, we used a damping factor of $\lambda_{L}=1.0$ for the lattice thermostat and a transverse damping $\lambda_{s}=0.1$ for the spin system. Before the thermostat was applied, atomic velocities were set so as to obtain an initial temperature of $300 \mathrm{~K}$ (or $10 \mathrm{~K}$ in the cases of the simulations with $\mathrm{T}<$ $300 \mathrm{~K})$. Applying the thermostat, thermal equilibrium was reached in all cases in a time between 2 ps and 10 ps with some fluctuations around the set temperature. Similarly, the resulting magnetization quickly reaches a stable value and remains stable during the simulation although its components may fluctuate, as shown in Fig. 2.

Once the simulation results were obtained, we conducted post-processing analyses using the free software OvITO [52]. In particular, we employed the coordination analysis tool to distinguish between atoms in the outer layer of the sphere (shell or surface) and the inner ones (core) to obtain the magnetization curves of these two different regions. In all cases, a single layer of atoms was selected within the surface group, as shown in Fig. 3. Details of the number of atoms contained in each NP and the ones belonging to the surface and the core regions are presented in Table I.

TABLE I. Total number of atoms, surface atoms, core atoms, and percentage of surface and core atoms for each studied Fe nanocluster.

Diameter No. of atoms Surface atoms Core atoms \% surface $\%$ core

\begin{tabular}{lccccc}
\hline $2 \mathrm{~nm}$ & 339 & 177 & 162 & 52.21 & 47.79 \\
$3 \mathrm{~nm}$ & 1243 & 528 & 715 & 42.48 & 57.52 \\
$4 \mathrm{~nm}$ & 2741 & 808 & 1933 & 29.48 & 70.52 \\
$5 \mathrm{~nm}$ & 5601 & 1480 & 4121 & 26.42 & 73.58 \\
$6 \mathrm{~nm}$ & 9577 & 2054 & 7523 & 21.45 & 78.55 \\
$8 \mathrm{~nm}$ & 22659 & 3695 & 18964 & 16.31 & 83.69 \\
\hline \hline
\end{tabular}

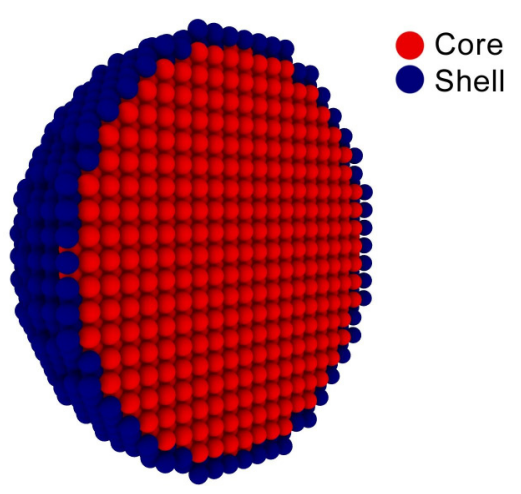

FIG. 3. Snapshot of one half of a 6-nm diameter Fe NP, obtained with software OVITO [52], inscribed in a cubic region with periodic boundary conditions. The shell and core regions are indicated by the colors, where each small sphere represents an atom.

Total magnetization averages were computed over the last 300000 steps of the simulation. To minimize computational cost, core, and shell magnetization $\left(\mathrm{M}_{c}\right.$ and $\mathrm{M}_{s}$, respectively) results were obtained averaging over the last $100 \mathrm{ps}$ of the simulation.

\section{MEAN-FIELD ISING MODELS}

Coupled MD-spin simulations are an advanced modeling tool that has never been applied to magnetic NPs. It is therefore useful to probe our results by comparing them to simpler and more commonly used models. In this section, two variants of the mean-field Ising model have been applied to NPs to perform that comparison.

\section{A. Spin model including surface effects}

Our results can be compared with a theoretical model that includes surface effects on the magnetization. This model, originally proposed by Mills [53], is known as semi-infinite Ising model with a free surface, i.e., basically a mean-field Ising model of a ferromagnet with a free surface. It is assumed that the spins are arranged in a lattice (bcc in this case) and that the spins in all lattice sites are given by $S_{i}= \pm 1$ where $S_{i}=+1$ means that spin $i$ is pointing along the positive $z$ direction and $S_{i}=-1$ means that spin $i$ is pointing in the opposite direction. In this model, the exchange coupling constant $J$ is the same for all nearest-neighbor (NN) pairs, except for the case of pairs at the surface of the ferromagnet where it is denoted by $J_{s}$. A layered structure is then obtained with the Hamiltonian being given by

$$
\mathcal{H}=-J \sum_{\langle i, j\rangle \notin S}^{N} \boldsymbol{\mu}_{i} \cdot \boldsymbol{\mu}_{j}-J_{S} \sum_{\langle i, j\rangle \in S}^{N} \boldsymbol{\mu}_{i} \cdot \boldsymbol{\mu}_{j} .
$$

Following the mean-field approach, the magnetization for the surface $m_{s}$ and for each successive layer $m_{1}, m_{2}, \ldots m_{n}$, are given by

$$
\begin{gathered}
m_{s}=\left\langle\mu_{i \in S}\right\rangle=\tanh \left(4 K_{S} m_{S}+K m_{1}\right), \\
m_{1}=\left\langle\mu_{i \in 1}\right\rangle=\tanh \left(4 K m_{1}+K m_{S}+K m_{2}\right),
\end{gathered}
$$




$$
m_{n}=\left\langle\mu_{i \in n}\right\rangle=\tanh \left(4 K m_{n}+K m_{n-1}+K m_{n+1}\right),
$$

where $K_{s}=\beta J_{s}$ and $K=\beta J$ with $\beta=1 / k_{B} T$ and $k_{B}$ is the Boltzmann constant. It is important to notice that $K$ and $K_{s}$ are not related to the anisotropy constants $K_{1}$ and $K_{2}$ described previously. In this paper, we have adopted a two-layer approximation, meaning that we have considered that the system's magnetization is unaltered after the first layer (the one after the surface), $m_{1}=m_{2} \cdots=m_{n}=m_{\text {bulk }}$. Therefore, $m_{1}$ represents the cluster core magnetization and, in addition, we have set $J_{s}=J$. In this way, the summation on the first term of the right-hand side of the Hamiltonian [Eq. 14)], runs over the eight first neighbors (core) and the one on the second term runs over four first neighbors [(100)-like surface]. Related electronic models have been used to obtain the magnetization of Fe thin films [44].

\section{B. Spin model including finite-size effects}

A previous theoretical model developed in Ref. [54] is summarized below. The model is based on the mean-field approximation for the Ising model, adapted to the statistics of few-particle systems. The mean-field approach is well known and does not need explanation. However, if a few-particle system is analyzed, we should be careful with the approximations that are used when obtaining the fundamental equations in the microcanonical formalism [55]. In particular, the Stirling approximation $(\ln x ! \approx x \ln x-x)$ cannot be applied, and the factorial must be written in terms of the Gamma function: $\Gamma(x)=(x+1)$ !. This implies working with the derivative of this function, known as the digamma function:

$$
\psi^{(0)}(x)=\frac{d \Gamma}{d x} .
$$

Taking this into account, a self-consistent equation for the magnetization $M$ is found. If there are $N$ atoms in the system and the coordination number between them is $z$, the magnetization is given by the solution of the equation

$M=\frac{k_{B} T}{z J}\left\{\psi^{(0)}\left[\frac{N}{2}(1+M)+1\right]-\psi^{(0)}\left[\frac{N}{2}(1-M)+1\right]\right\}$.

Naturally, this simple model does not allow us to make precise quantitative predictions due to the coarseness of the mean-field approach. However, one could expect to obtain a reasonable qualitative comparison with the results of the simulations. Equation (19) has to be solved numerically for the conditions in each simulation.

\section{RESULTS AND DISCUSSION}

\section{A. Simulation results}

Typical initial atomic and spin configurations and its evolution after 500 ps of simulation can be seen in Figs. 4 and 5 for different NP sizes and temperatures. For more insight about the individual atomic spins dynamics, the reader is referred to movies SM1 and SM2 in the Supplemental Material [51], where evolution of simulations are shown for a group of spins
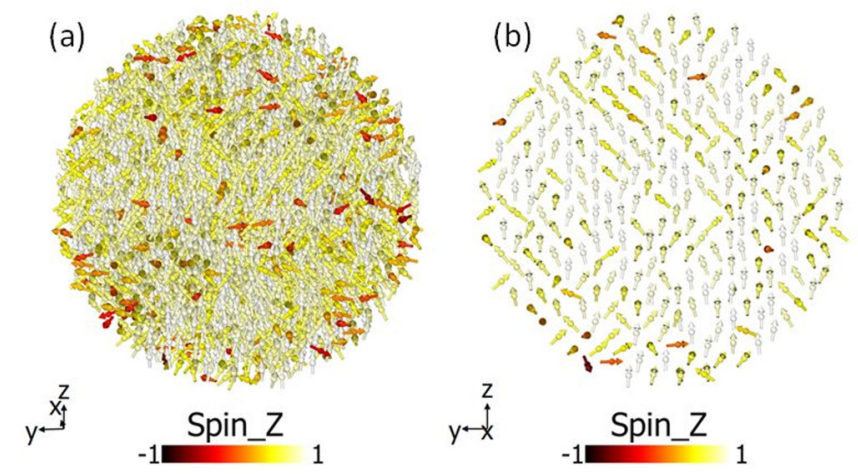

FIG. 4. Snapshots of a typical simulation of a NP with a diameter of $6 \mathrm{~nm}$. In (a), the atomic spins are displayed as arrows, with color indicating magnitude of $m_{z}$ at $600 \mathrm{~K}$. (b) Same as (a) for thin slab at the center of the nanoparticle. The initial condition of the simulations was $m_{z}=1$, i.e., all spins pointing along the positive $z$ axis. See also the movies SM1 and SM2 in the Supplemental Material [51].

belonging to a slab at the center of the sphere (SM1) and also for the spins from the surface of the NP (SM2).

The temperature dependence of the total magnetization is shown in Fig. 6 for NPs of 2, 4, and $8 \mathrm{~nm}$ in diameter, along with the bulk system. Results for all simulated clusters are not included in this graph to avoid cluttering; a graph with the complete set of the simulated NPs appears in Fig. S2 of the Supplemental Material [51]. Figure 6 indicates that the bulk Curie temperature, $T_{C}$, in our simulations is around $650 \mathrm{~K}$, lower than the $1040 \mathrm{~K}$ in experiments, and this difference

(a)

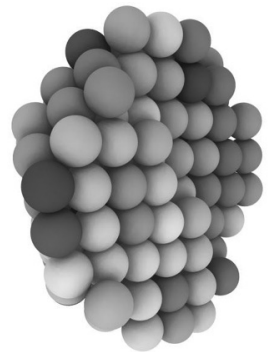

(c)

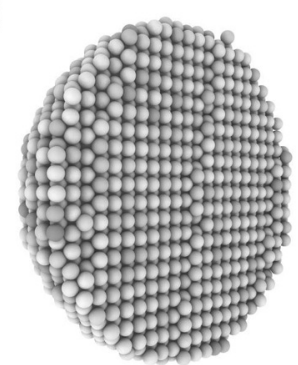

(b)

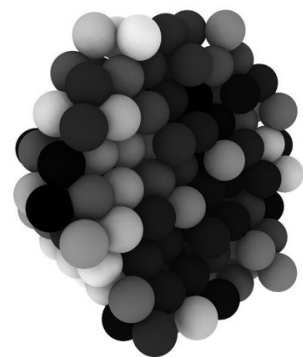

(d)

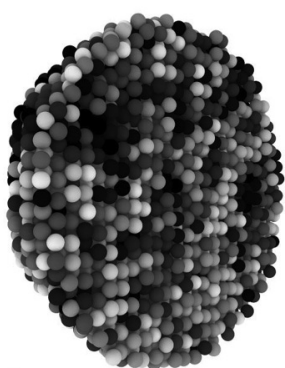

Magnetization along ' $z$ ' axis

FIG. 5. Snapshots of simulations showing the z-component $m_{z}$ of the atomic magnetization for nanoparticles having (a) $2 \mathrm{~nm}$ and $300 \mathrm{~K}$, (b) $2 \mathrm{~nm}$ and $1200 \mathrm{~K}$, (c) $6 \mathrm{~nm}$ and $300 \mathrm{~K}$, and (d) $6 \mathrm{~nm}$ and $1200 \mathrm{~K}$. The small spheres represent atoms. White indicates that the spins point along the positive $z$ axis, while black indicates that $m_{z}$ points along the negative $z$ axis. The $z$ axis is vertical with the positive direction pointing upward in the figures. 


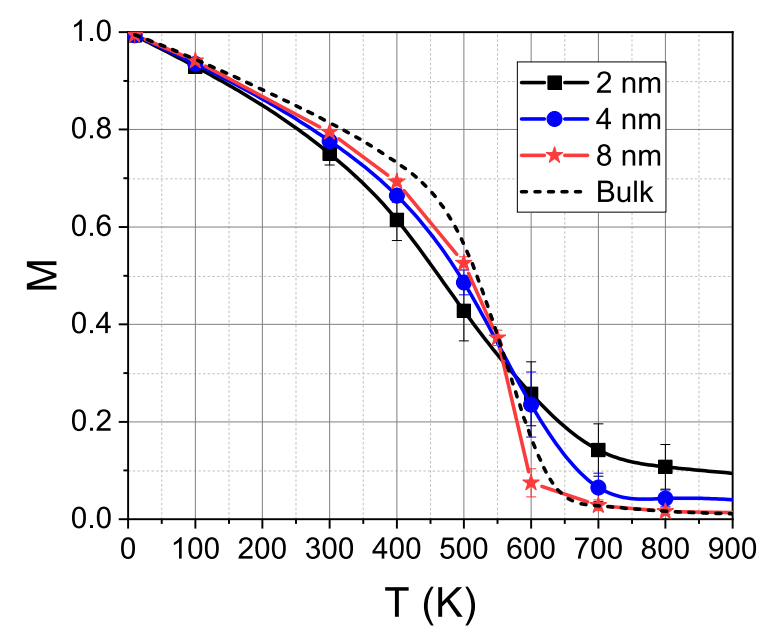

FIG. 6. Total normalized magnetization as a function of temperature for different NPs compared to the bulk values.

is discussed later. From Fig. 6, there are indications of an increase of $T_{C}$ with decreasing cluster size, reaching around $T_{C} \sim 800 \mathrm{~K}$ for a 2 -nm cluster with $\sim 500$ atoms.

At the low-temperature regime, as the size of the Fe nanoclusters is reduced, the magnetization takes lower values than the bulk ones for all sizes. The difference is larger as the temperature is increased up to about $550-600 \mathrm{~K}$. This is the expected behavior since the surface contribution to the total magnetization is more important in smaller NPs due to the larger surface/bulk proportion (see Table I). When the temperature is raised to about $T \approx 500 \mathrm{~K}$, the surface magnetization decreases faster than the core contribution since the spins on the outer layer, having smaller local coordination number, are disordered more easily (see Fig. 11). For temperatures higher than $500 \mathrm{~K}$, the bulk magnetization is the one that decreases faster, i.e., changing to the opposite behavior. This is displayed by the interception of the curves around $\mathrm{T} \approx$ $550 \mathrm{~K}$ in Fig. 6. A similar crossover has been also observed in other studies of magnetic NPs [50,56].

It is interesting to compare our results with those of simulations in which the atoms are static. In Fig. 7, we have compared the magnetization curves with the ones corresponding to NPs with fixed atomic positions. At low temperatures, below $400 \mathrm{~K}$, there is no significant difference. Our results for bulk Fe are consistent with other simulations which found only a small decrease of the critical temperature with the inclusion of a thermalized, moving lattice [36,57]. For the 2-nm case, the moving atoms lead to a minor decrease in magnetization, since the NP is already quite disordered, from a magnetic point of view, at temperatures where the fluctuations of the interatomic distances become important due to the large fraction of surface atoms (see Table I). For the 8-nm case, magnetization goes to zero at lower temperatures for the moving atoms, as expected from the bulk simulations. At $600 \mathrm{~K}$, near the critical temperature, the mean value of the NN distances changes less than $1 \%$ with respect to the one for the frozen lattice. As a result, the value of $J\left(r_{i j}\right)$ averaged over the distribution of $\mathrm{NN}$ atomic distances at $600 \mathrm{~K}$ is only slightly different than the value $J\left(r_{\mathrm{NN}}\right)$ for fixed atoms, changing less than $\sim 3 \%$. Therefore, neither a lattice expansion nor
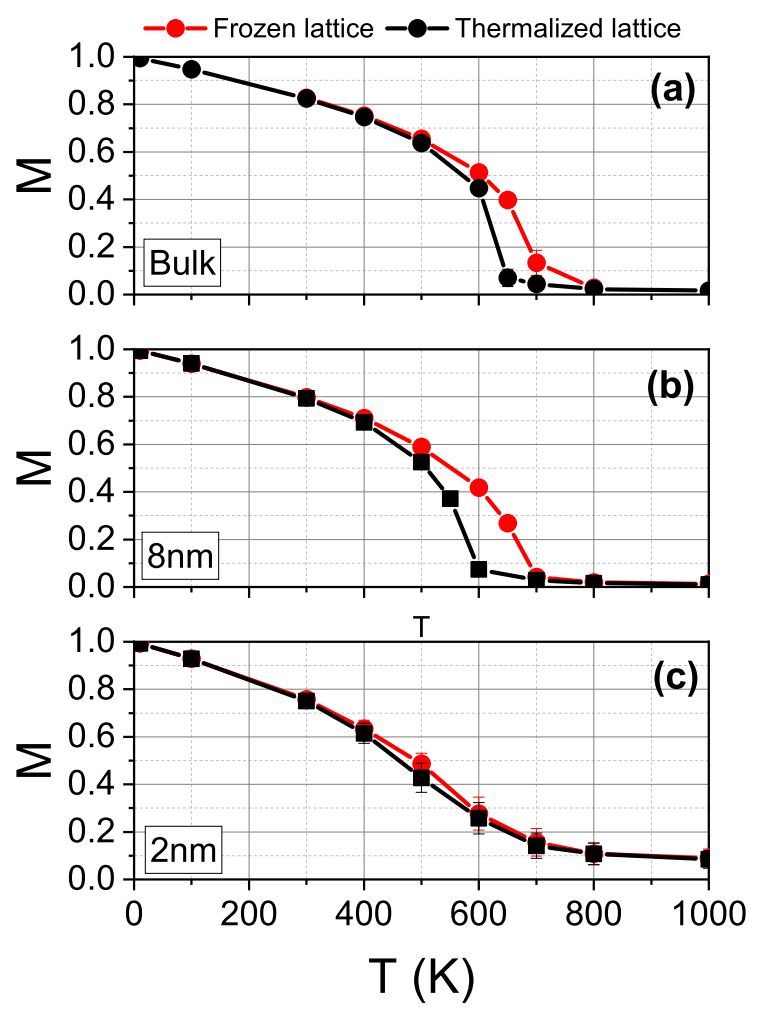

FIG. 7. Temperature dependence of the magnetization $M(T)$ as obtained using MD-SD, for (a) Fe bulk, nanoparticle diameters (b) $8 \mathrm{~nm}$, and (c) $2 \mathrm{~nm}$. The results for thermalized and frozen translational degrees of freedom are compared. Bulk results correspond to cubic simulation boxes with $20 a_{0}$ sides.

a fluctuating mean value for $J$ can explain the decrease in magnetization found in our simulations for a moving lattice. This is a clear indication that the coupling between lattice and spins play a significant role for not-too-small $\mathrm{Fe}$ particles near $T_{C}$. Calculation of time and spatial correlation functions might help understanding this in detail.

Complementary insight is obtained by comparing our results with simulations for static spins in a regular lattice [58]. Let us assume only NN interactions and $J=$ $15 \mathrm{meV}$, similar to the value in our simulations for distances between first and second neighbors. The Ising model in $3 \mathrm{D}$ gives $T_{C}=z J / k_{B}$ for the mean-field approximation, where $z$ is the coordination number ( 8 for bcc) resulting in $T_{C}=1393 \mathrm{~K}$. However, Bethe's solution gives $T_{C} \approx$ $2 J / k_{B} \ln (z /(z-2)) \approx 1200 \mathrm{~K}$. The classical Heisenberg model gives $T_{C} \approx 4 z J / 3 k_{B}=1857 \mathrm{~K}$, using the mean-field approximation. Using a high-temperature expansion, this changes to $(105 / 96)(z-1) J / k_{B}=1333 \mathrm{~K}$ [58]. As expected, the mean-field approximation overestimates the Curie temperature compared to the exact analytical models or to numerical solutions.

Our NPs magnetization results are consistent, and show really good agreement, with the experimental magnetization curves for $\mathrm{Fe}$ nanoclusters reported by Billas et al. [59], Fig. 8(a), where it can be seen that both the shape of the curves and the estimated Curie temperature for the iron nanoclusters are well reproduced. To compare with experimental results, we have assumed a constant local magnetic moment of $2.2 \mu_{B}$. 

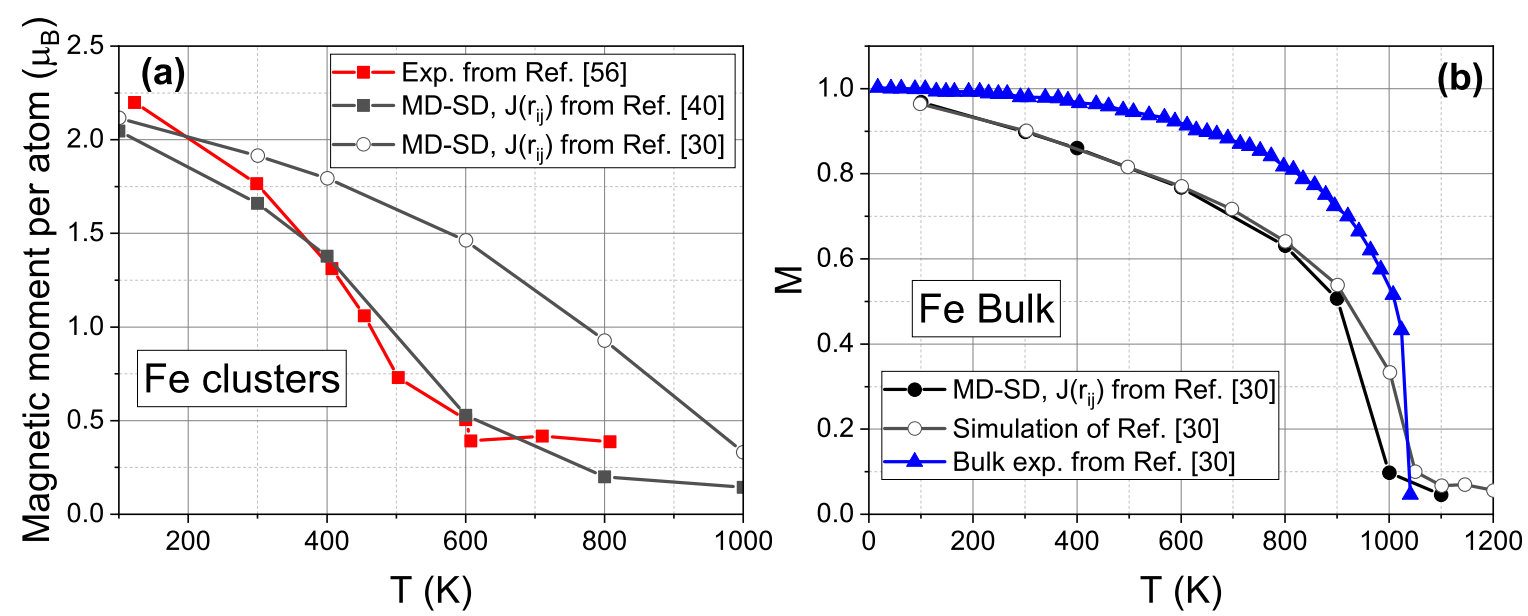

FIG. 8. (a) Experimental results for a Fe nanocluster of about 500-600 atoms as reported by Billas et al. [59] compared to the results of our simulations of a Fe NP composed of 533 atoms using two different exchange functions $J\left(r_{i j}\right)$. The exchange function $J\left(r_{i j}\right)$ fitted from the calculations of Pajda et al. [40] is the one used in this work and it reproduce quite closely the experimental results. The open circles correspond to simulation results using the exchange function proposed by Ma et al. [30]. (b) Magnetization of a Fe Bulk system (cell size $20 \times 20 \times 20 a_{0}^{3}$ ) as obtained using the exchange function proposed by Ma et al. [30] (full circles). Bulk experimental data (triangles) and the results reported by Ma et al. (open circles) are also shown for the sake of comparison.

While our simulation results match with experiments for small clusters, our bulk simulations yield an estimated Curie temperature around $T_{C} \approx 650 \mathrm{~K}$, far below the experimental value of $1043 \mathrm{~K}$. The main reason for this discrepancy is the exchange coupling function used in this paper. When $J\left(r_{i j}\right)$ is replaced, for example, by the one used by Ma et al. [30] (note the large discrepancies found in the literature for $J(r)$ reported in Fig. 1 of Ref. [30]), our simulations show similar results regarding the experimental Fe Curie temperature, as the results reported in that paper [Fig. 8(b)]. Should one use those parameters for small clusters, one obtains an overestimation of the cluster $T_{C}$. Hypothetically, this is an indication of an important increase of the effective exchange function $J\left(r_{i j}\right)$ as the size of the NPs increase. It also reflects the challenge of modeling broad size ranges in the size-dependent effective interaction parameters.

Some recent SD simulations which incorporate finitetemperature effects obtain a lower Curie temperature, $T_{C}$, than experimental values $[36,60]$. It is known that SD simulations tend to smooth the ferromagnetic-paramagnetic transition near the Curie temperature due to the intrinsic coarse-graining of the numerical scheme and since quantum effects are not taken into account [20]. Nevertheless, adding a quantum mechanical treatment does not guarantee a correct determination of $T_{C}$ [61]. The discrepancy between $T_{C}$ obtained in our simulations and the experimental one could also be partly related to the fact that our magnetic exchange function $J\left(r_{i j}\right)$ is not temperature nor size sensitive. In fact, in this framework, it is not possible to take into account any temperature-dependence changes in the electronic structure from which $J\left(r_{i j}\right)$ is obtained. Modeling an exchange parameter that is function of $T$ is expected to lead to a more precise estimation of $T_{C}$ $[20,62,63]$. In addition, including system-size variations of $J\left(r_{i j}\right)$ might help reproducing the changes in $T_{C}$ which can be derived from $\mathrm{Fe}$ cluster experiments [59].

Figure 9 displays the size dependence of the total magnetization $M(T)$ which is plotted as a function of the inverse diameter for representative temperatures $T$. This allows us to show the bulk magnetization values corresponding to $1 / d=0$. At low temperatures, the calculated size dependence is almost negligible. This trend contrasts with the well-known enhancement of the average ground-state magnetization in $\mathrm{Fe}$ clusters and surfaces, which can be qualitatively explained from an electronic perspective as a consequence of $3 d$-band narrowing [64]. The effect could be easily incorporated in our simulation by taking into account the size dependence of

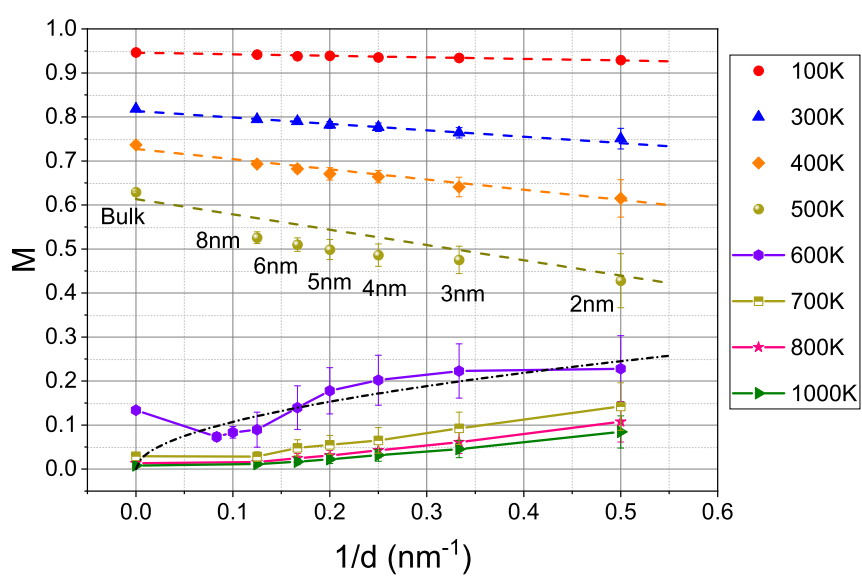

FIG. 9. Size dependence of the total magnetization at different representative temperatures. The normalized magnetization is plotted as a function of the particle inverse diameter $(1 / d)$. Symbols and full curves correspond to the simulation results, whereas the dashed lines represent the fitted curves. The error bars indicate the standard deviation of the data that are taken into account in the average over the last $0.3 \mathrm{~ns}$. For the fitted curves, the function is $M=a(1-$ $\left.b^{T}\right)(1 / d)+\left(\frac{T-T_{C}}{T}\right)^{1 / 3}$ where $a=0.1, b=1.003$, and $T_{C}=650 \mathrm{~K}$. The black dash-dotted line corresponds to $M=0.35(1 / d)^{0.514}$ and is related to the magnetization scaling behavior near the critical temperature in the 3D Heisenberg model as detailed in the text. 
the local magnetic moment $\mu_{i}=\left|\left\langle s_{i}\right\rangle\right|$. As the temperature increases, so does the influence of the NP size on the total magnetization. These results are in good agreement with those reported by Iglesias and Labarta [7], who studied the size effects on maghemite NPs via Monte Carlo simulations.

It is notable that Fig. 9 clearly shows two different regimes: low $(T<600 \mathrm{~K})$ and high $(T>600 \mathrm{~K})$ temperatures, separated by the $600 \mathrm{~K}$ curve. For temperatures below $600 \mathrm{~K}$, we find that the magnetization behavior is almost linear with the inverse diameter, showing higher values for larger NPs. This trend is expected, as adding more magnetic atoms to the particle stabilizes a stronger ferromagnetism, and thus increases the resilience of the total magnetization to thermal disorder. In addition, this linear size dependence is stronger when the temperature is increased. To further analyze these results, we have proposed a functional with only two free parameters for the magnetization as a function of temperature and cluster size. This approximation manages to reproduce the size dependence of the magnetization very well for temperatures below $600 \mathrm{~K}$, as can be seen from the dashed lines in Fig. 9. The corresponding function is

$$
M(T, d)=a\left(1-b^{T}\right) \frac{1}{d}+\left(\frac{T_{C}-T}{T_{C}}\right)^{1 / 3},
$$

with $a=0.1, b=1.003, T_{C}=650 \mathrm{~K}$, and $d$ the NPs diameter.

For temperatures above $600 \mathrm{~K}$, the linear relation between $M(T)$ and $1 / d$ is reversed, although the trend remains almost linear. At higher $T$, larger NPs retain less magnetization than smaller ones. This qualitative change reflects the intersection of the magnetization curves in Fig. 6.

The two different regimes observed in Fig. 9 are separated from each other by the $600 \mathrm{~K}$ curve, where a different trend is observed. This different behavior may be due to competing effects, as the system is close to the critical temperature.

Behavior of the magnetization with varying size is governed by finite-size scaling laws. According to finite-size scaling theory $[66,67]$, magnetization near the critical point should scale with size as $M \approx L^{(\beta / v)}$, where $\beta$ and $v$ are the critical exponents related to the order parameter and correlation length, respectively. This means that the value of the magnetization for the bulk near the critical point would be lower than the simple extrapolation from a linear fit, as the one shown in Fig. 1. Magnetization near the critical temperature is expected to scale as $M(1 / L)^{(\beta / v)}=(1 / L)^{0.514}$, where $\beta / \nu=0.514$ is the estimated value for the critical exponents of the 3D Heisenberg model [68-71]. Therefore, the bulk value should be closer to the values for finite size NPs, as also shown in that figure. In addition, the behavior of the $600 \mathrm{~K}$ curve for clusters is also consistent with these arguments, as shown by the dashed-dotted line in Fig. 9, corresponding to $M=0.35(1 / d)^{0.514}$

In practice, Fig. 9 shows that even above $T_{c}$, small subclusters of atoms with similar spin orientations nucleate in the NPs as well as in the bulk cells (before rapidly dissipating). In large NPs and bulk cells, those local clusters are likely to be averaged down by the larger number of atoms, or by other local clusters with opposition magnetization. As NPs are getting smaller, fewer clusters can nucleate and thus cannot average

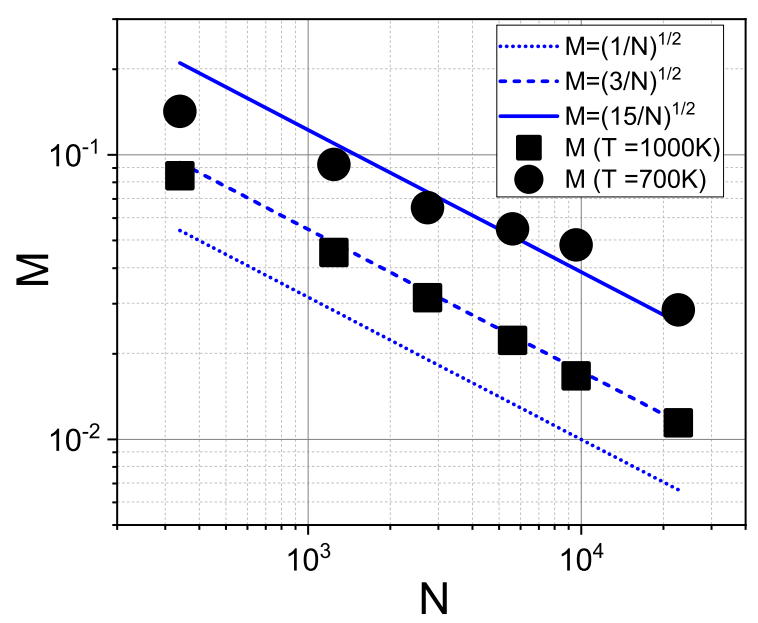

FIG. 10. Fe NP magnetization as a function of the number of atoms $N$ in the nanoclusters (symbols) at two representative temperatures above the predicted cluster $T_{C}$, compared with different power law relations, including the one proposed in Ref. [65], for clusters with 15 atoms.

down the resulting local magnetic moments of each others. This results in the persistence of a small net magnetization at higher temperature. The trend observed above $600 \mathrm{~K}$ may be thus explained by the presence of spin-spin correlations above the Curie temperature. Indeed, short-range magnetic ordering (SRMO) is known to persist in the paramagnetic state of iron bulk and surfaces [72-74]. The importance of SRMO in Fe above the Curie temperature has been explicitly demonstrated in Ref. [65], where it was shown that correlated clusters with size $N_{c l}$ for a system with $N$ atoms led to a magnetization $M \propto$ $\left(N_{c l} / N\right)^{1 / 2}$, with $N_{c l}=15$ for Fe. This dependence is similar to the one above from finite size-scaling, and $N_{c l}=15$ is consistent with 1st and 2nd NN shells in the bcc lattice, totalling 14 atoms, which is expected since the $2 \mathrm{nd} \mathrm{NN}$ shell is only around $14 \%$ further away than the $\mathrm{NN}$ shell, as it is shown later in this section. Fig. 10 shows that our MD simulations near $T_{C}$, at $700 \mathrm{~K}$, follow this relationship extremely well, and that for larger temperatures $N_{c l}$ decreases significantly, due to thermal fluctuations decreasing magnetic correlation as expected. In future work, the temperature dependence of spin-spin correlation functions and related structure factors could be evaluated for different NP sizes in order to study this in detail.

The two different regimes observed in Fig. 9 may also be explained by assuming distinct core and surface magnetizations. Making this distinction, the total magnetization can be written as $M(T)=M_{c}(T)-\left[M_{c}(T)-M_{s}(T)\right] \frac{1}{d}$, where $M_{c}(T)$ and $M_{s}(T)$ represents, respectively, the core and shell magnetization contributions for a NP of diameter $d$. In this way, the total magnetization is closer to the bulk magnetization as the size of the NP increases. Consequently, if $M_{s}(T)$ decreases faster than $M_{c}(T)$ as the temperature is increased, the slope of $M$ vs. $(1 / d)$ becomes steeper, $\left(\left[M_{c}(T)-M_{s}(T)\right]\right.$ becomes larger), which is the case observed in Fig. 9 until $T=500 \mathrm{~K}$. At $600 \mathrm{~K}$, for the smaller NPs $\left(\frac{1}{d} \geqslant 0.33\right)$, both the interior and surface spins are disordered, that is, $M_{c}(T) \simeq$ $M_{s}(T)$ and the curve is flatter, i.e. practically does not depend 


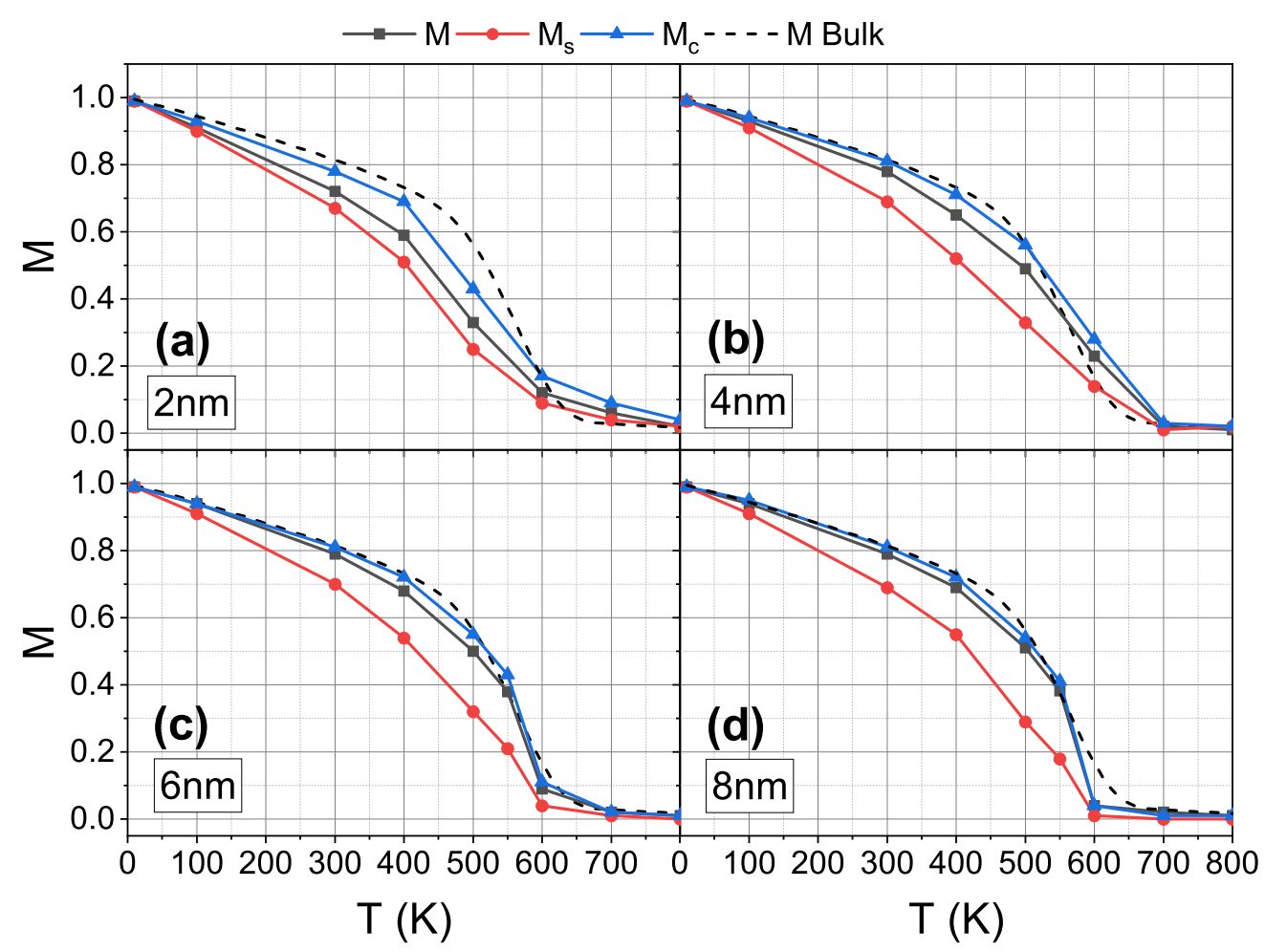

FIG. 11. Total normalized magnetization $M$, core magnetization $M_{c}$ and shell magnetization $M_{s}$ a functions of temperature for Fe NPs having different diameters. The Fe bulk magnetization is also shown.

on $1 / d$. For larger nanoclusters, both shell and core spins are disordered, resulting in a small global magnetization.

Having a free surface on the NPs naturally introduces finite-size effects on the thermal behavior of the magnetization. The effects of the free surface were studied for different NPs and results are shown in Fig. 11. Surface (red dots) and core (blue triangles) contributions to the total magnetization (black squares) are plotted along with bulk magnetization results (dashed line). Surface magnetization is calculated averaging over the atoms belonging to a single-atom wide spherical shell composed of the outer layer of atoms as described in sub-section (II-B) (see Fig. 3).

The width of the surface is held constant for all spheres. The main qualitatively result is that the NPs retain less magnetization than the bulk system and this difference in magnetization increases as the particle size decreases. This is a consequence of the low coordination of the spins at the surface, together with the large surface-to-volume ratio and their contributions to the total magnetization. In this way, it is clear that for the $2 \mathrm{~nm}$ sphere the surface contribution is as relevant as the core one. Therefore, all three curves, $M, M_{s}$ and $M_{c}$, are far apart from the bulk values. As the particle diameter increases, the surface contribution to the total magnetization decreases. Thus, two main features can be seen: the total magnetization becomes increasingly similar to the core magnetization and, straightforwardly, the core magnetization approaches more and more the bulk magnetization values. This tendency of the core magnetization to approach to the bulk values is stronger at low temperatures, while near $T_{C}$ there is still a clear departure from the bulk behavior. In addition, there is a temperature range where the cluster magnetization behavior and, in particular, the surface contribution displays an approximately linear temperature dependence, as it has been previously reported by Iglesias and Labarta [7]. Furthermore, this temperature range is larger for smaller particles. In Ref. [7], it was argued that this linear behavior was related to an effective 3D-2D dimensional reduction of the surface shell, and that it has been observed in thin film systems and in simulations of rough ferromagnetic surfaces [75-79].

The surface, core and total magnetization are related by

$$
M=p M_{s}+(1-p) M_{c},
$$

where $p=N_{s} / N_{T}$ is the shell fraction, $N_{S}\left(N_{T}\right)$ being shell (total) number of atoms [7]. Assuming that the shell is much thinner than the NP radius, one can arrive at the approximate expression

$$
M(d)=M_{c}-\Delta M \frac{\Delta r S}{V}=M_{c}-\Delta M \frac{6 \Delta r}{D},
$$

where $S(V)$ are the surface (volume) of the particle, $\Delta r$ is the thickness of the surface layer, $D$ is the diameter of the spheres and $\Delta M=M_{c}-M_{s}$. Figure 12 displays a good agreement between the simple two component approximation and our simulation results. In particular, Eq. (22) manages to reproduce the intersection of the curves around $T_{C}$, a fact that is related to the surface/core proportion as discussed above.

\section{B. Exploring possible NP premelting}

The EAM potential used in the previously mentioned study yields a bulk melting temperature $T_{M} \simeq 2000 \mathrm{~K}$ [80]. However, small clusters are expected to have much lower $T_{M}$ due to the reduced surface coordination number. Indeed, in Ref. [81], a reduction of about $30 \%$ in the melting temperature of 2-nm 


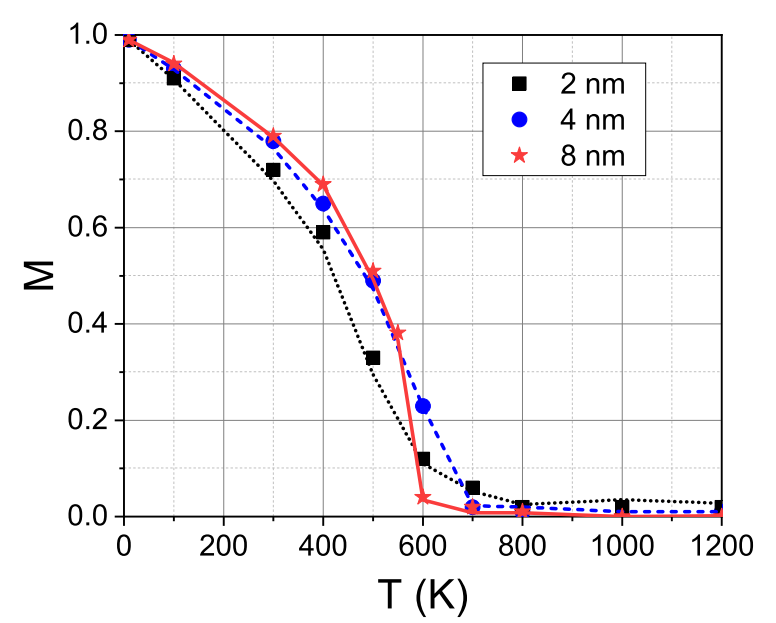

FIG. 12. Temperature dependence of the total magnetization $M$ of Fe NPs.The results of our MD-SD simulations (symbols) are compared with the core-shell two component model described by Eq. (22) (curves).

Fe clusters has been reported. Assuming the same reduction of $T_{M}$ for the EAM potential used here would give $T_{M} \simeq 1350 \mathrm{~K}$. The structure and diffusivity of core and shell atoms have been analyzed at different temperatures, to evaluate possible premelting of $\mathrm{Fe}$ nanoclusters in the framework of our model. The pair correlation function, $g(r)$, for the whole nanocluster, is shown in Fig. 13. There are broad but well defined peaks for second- and third-NNs at high temperatures, as expected for a crystalline solid.

At $1200 \mathrm{~K}$, the NN distance for bulk Fe is $0.245 \mathrm{~nm}$, while for the smallest NPs this distance is closer to $0.25 \mathrm{~nm}$, which correpond to a change of only $2 \%$. The effect of large changes in neighbor distance for Fe films was evaluated by GaribayAlonso et al. [44], who also observed a nearly linear decrease of the layer magnetization with temperature, as in Fig. 11. The radial distribution functions $g(r)$ shown in Fig. 14 for
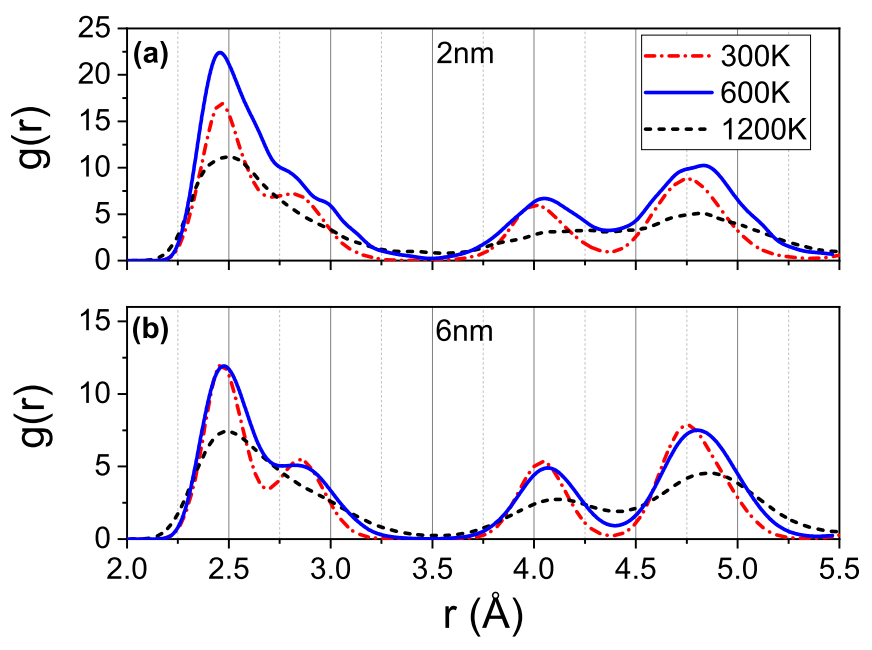

FIG. 13. Coordination analysis for spherical Fe NPs with diameters (a) $d=2 \mathrm{~nm}$ and (b) $d=6 \mathrm{~nm}$ at different temperatures. The data are taken from the last configuration of the system. They indicate lack of melting, even for the smallest considered nanocluster.

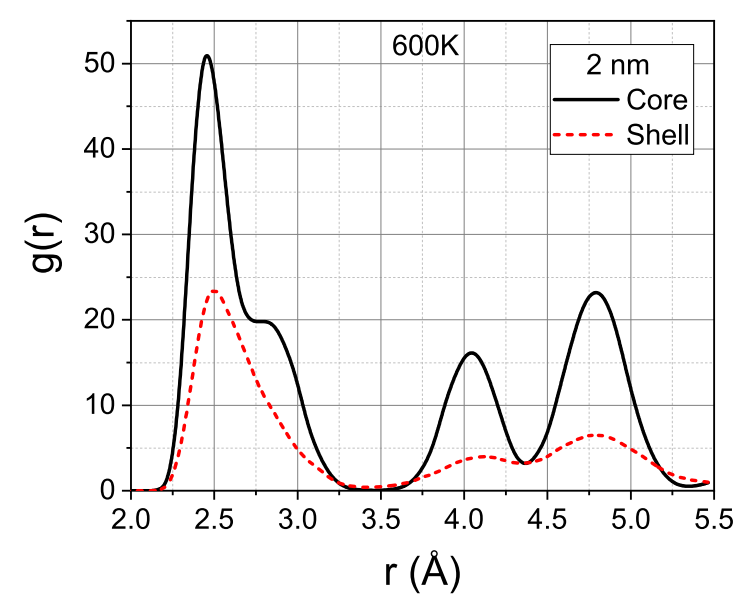

FIG. 14. Correlation function in the core and shell regions of a $2 \mathrm{~nm} \mathrm{Fe} \mathrm{NP}$ at $T=1200 \mathrm{~K}$.

$T=600 \mathrm{~K}$, show that there are non-negligible fluctuations on the first and second-neighbor separation distance as the size of the system is reduced. These fluctuations may result in values of $J\left(r_{i j}\right)$ (see parametrization of $J\left(r_{i j}\right)$ in Ref. [37]), that could drive the systems into an antiferromagnetic phase for the smallest clusters at $600 \mathrm{~K}$, as it is shown in Ghosh et al. [82].

In Fig. 14, results are given for the interatomic correlation function $g(r)$ in the shell and core regions at a temperature near the cluster $T_{C}$. The well-defined peaks in $g(r)$ show no evidence of surface premelting at this temperature, even for the smallest NPs. To further investigate this mater, we have carried out atomic diffusivity calculations. At 600 and $1200 \mathrm{~K}$, diffusivity for core atoms has a value close to zero, but for shell atoms has values of the order of $D=4.0 \times 10^{-10} \mathrm{~m}^{2} / \mathrm{s}$, as seen in Fig. 15. For reference, the bulk self-diffusion coefficients values are around $D=1.0 \times 10^{-15} \mathrm{~m}^{2} / \mathrm{s}$ for $1000 \mathrm{~K}$ as reported in Ref. [83]. The diffusivity for molten

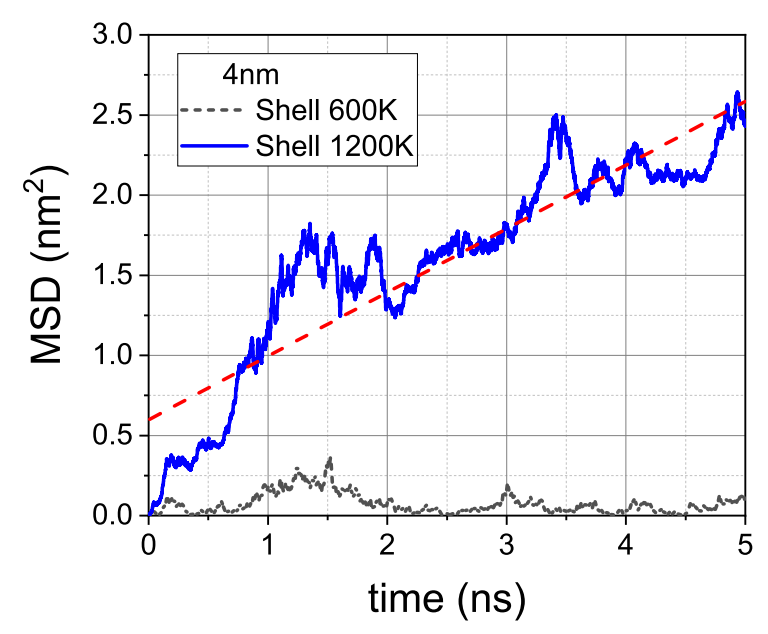

FIG. 15. Mean-square displacement of the shell atoms at 600 and $1200 \mathrm{~K}$, as obtained from our simulations for a $4 \mathrm{~nm} \mathrm{NP}$. The straight dashed line represents a linear fit of the $1200 \mathrm{~K}$ results, giving a diffusion coefficient $D=4.0 \times 10^{-10} \mathrm{~m}^{2} / \mathrm{s}$. Note that this simulation is 10 times longer (5 ns) than those performed for the magnetization calculations. 

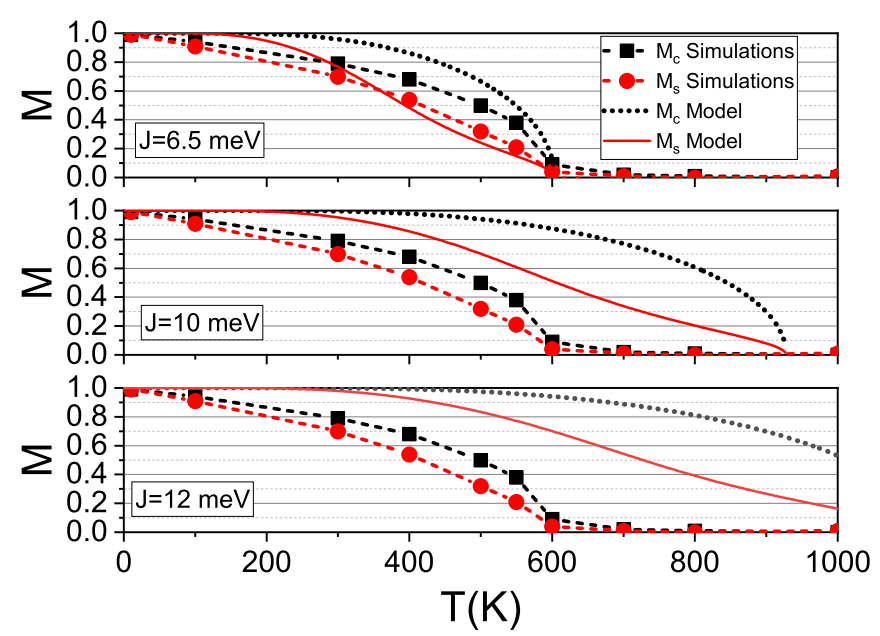

FIG. 16. Temperature dependence of the core and shell magnetizations as obtained from the semi-infinite Ising model with a free surface for different values of the exchange constant J (Sec. III A) compared with MD-SD simulations results. The curves are obtained for a Fe NP with a diameter of $6 \mathrm{~nm}$.

Fe has been typically calculated above $1500 \mathrm{~K}$. For instance, Gosh et al. [84] used the EAM parameters of Bhuiyan et al. [85] and obtained values which, extrapolated down to $1230 \mathrm{~K}$ give $D$ close to $1.5 \times 10^{-9} \mathrm{~m}^{2} / \mathrm{s}$. Given that, according to the results shown in Fig. 15, the shell region of a 4-nm Fe NP at $1200 \mathrm{~K}$ has a diffusivity similar to extrapolated molten bulk values, one could argue that the shell region is molten or partially molten at $1200 \mathrm{~K}$, while at $600 \mathrm{~K}$ it is still solidlike. However, we note that the shell region contains mostly surface atoms, and that surface diffusion is complex at high temperatures [46]. Therefore, further studies are needed to elucidate this point.

\section{Comparison with theoretical models}

We have compared our simulation results with a semiinfinite mean-field Ising model, an analytical model that includes surface effects and is described in Sec. III A. In Fig. 16, we have compared the bulk and shell magnetizations obtained from the model with the ones from MD-SD simulations for different values of the exchange constant $J$. As can be seen, the model results are highly sensitive to the value $J$, but the qualitative behavior of the system is reasonably well reproduced for the three values considered. The thermal behavior of the analytical surface magnetization reproduces the shape of the corresponding MD-SD curve, also displaying an inflexion point at high temperatures, i.e., $d M / d T$ does not show a monotonous decrease. The rapid decrease of surface magnetization is also observed for the surface magnetization of thin films [44].

Figure 17 shows the analytical results of the model presented in Sec. III B and the computational results for different NPs, showing a good qualitative agreement. It can be seen that the rough qualitative behavior of the different magnetizations is well reproduced.

Quantitative agreement is difficult to achieve using meanfield models like the ones in Figs. 16 and 17. As expected, any

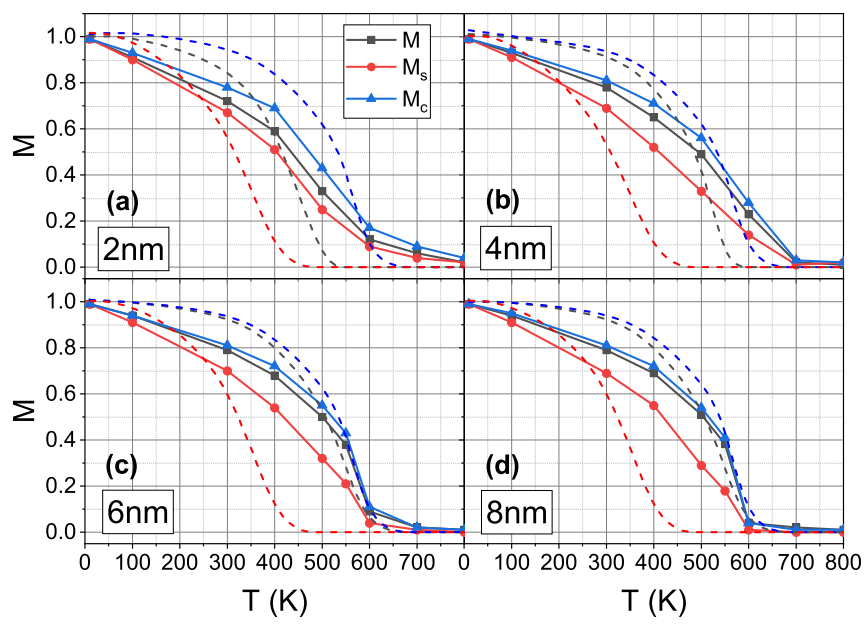

FIG. 17. Total, core and shell magnetization curves $M, M_{c}, M_{s}$, as obtained in the model presented in Sec. III B (dashed lines) [54] and in the present MD-SD simulations (solid line and symbols) for Fe NPs having different diameters.

mean-field model requires an effective $\mathbf{J}$ much lower than the one used in our spin Hamiltonian to adjust the critical temperature. Both models show good agreement with our results for $J=6.5 \mathrm{meV}$. In addition, there are non-negligible differences between Ising and Heisenberg models, as expanded below. Nevertheless, these results are usefull in order to test and support the MD-SD results. It is notable how the model manages to reproduce very well the behavior of the different magnetizations as the size of the NP is enlarged. It shows, as well as the MD-SD simulations, that an $8 \mathrm{~nm}$ diameter nanosphere behaves closely to a bulk system.

The Ising model variations shown above do not offer an accurate quantitative prediction of our magnetization curves. All of them use the mean-field approximation, and only up/down spin states. This is because solving the Heisenberg model in $3 \mathrm{D}$ is not trivial, even for periodic boundary conditions. Recent work shows the "phase diagram" for different values of $J$ at first-, second-, and third-NNs $\left(J_{1}, J_{2}, J_{3}\right)$ [82]. The frontier for the $(\pi, \pi, \pi)$ antiferromagnetic phase appears at $J_{2} / J_{1}=2 / 3 \sim 0.67$. In our case, for the chosen $J\left(r_{i j}\right)$, this ratio changes with temperature, and it is also affected by strain in the NP but is close to $J_{2} / J_{1}=0.6$, and $J_{3} / J_{1}=0$. This puts our system in the ferromagnetic state and, therefore, close to the frontier between the $(0,0,0)$ ferromagnetic and $(\pi, \pi, \pi)$ phases. The (q,q,q) spiral phase is close, but $J_{3}>0$ would be needed to reach that region of the phase diagram. At $10 \mathrm{~K}$, the separation distance between NN spins $\left(d_{\mathrm{nn}}\right)$ is around $d_{\mathrm{nn}}=0.245 \mathrm{~nm}$, and the distance between second NN $\left(d_{2 \text { nd }}\right)$ is about $d_{2 \text { nd }}=0.285 \mathrm{~nm}$, resulting in $J_{1}=19.5 \mathrm{meV}$ and $J_{2}=12.12 \mathrm{meV}, J_{3}=0$, giving $J_{2} / J_{1}=0.62$. At $1000 \mathrm{~K}$, $d_{\mathrm{nn}}=0.25 \mathrm{~nm}, d_{2 \mathrm{nd}}=0.29 \mathrm{~nm}, J_{1}=19 \mathrm{meV}, J_{2}=11 \mathrm{meV}$, $J_{3}=0$, giving $J_{2} / J_{1}=0.58$.

As an alternative simple model for magnetization vs temperature, Fig. S3 of the Supplemental Material [51] shows the curve for the "shape" model by Kuz'min [86]. The comparison of the model with our bulk results shows reasonable agreement if one sets the critical temperature to $T_{c}=650 \mathrm{~K}$ in the model. 


\section{CONCLUSIONS}

We have performed combined MD and SD simulations on isolated bcc Fe spherical nanoclusters and studied their magnetic properties as a function of temperature and cluster size, in the absence of external magnetic fields. To this aim, we have used a classical spin Hamiltonian coupled to classical MD. The effect of anisotropy is also considered. Our results naturally include lattice expansion, surface stress, and other factors, which are difficult to include in spin lattice models. Our simulations include Fe nanoclusters with up to 23000 atoms, and bulk simulations with up to 250,000 atoms in the simulation cell. The temperature of the lattice and the spins can be defined without any additional scaling factors [37], as usually required in SLD simulations [24].

Given the complexity of solving 3D magnetic models, simulations including thermal lattice effects, like thermal expansion and lattice strain due to surface effects, can be valuable tools in understanding and predicting the behavior of nanoscale magnetic systems. We find significant differences between our simulations with moving atoms, and simulations with frozen atoms as in most atomistic SD simulations, specially at temperatures above $2 / 3$ of the critical temperature. Recently, lattice relaxation was found to produce significantly larger coercitivity enhancement in NPs than the case of an unrelaxed, fixed lattice [87].

Our results show excellent agreement with experimental measurements of Fe nanoclusters [59]. The magnetization thermal behavior of small clusters is well reproduced and the estimated Curie temperature is also very similar. Total magnetization decreases with increasing temperature and decreasing size and, therefore, the decrease of magnetization with temperature is faster for the smallest clusters. Qualitatively, these results are expected, but they are quantitatively different from the ones in simple semi-analytical mean-field Ising models, even when size and surface effects are considered. Above the Curie temperature we find that magnetization scales with system size as predicted by models assuming SRMO [65].

For temperatures above $1000 \mathrm{~K}$, we observe evidence of possible melting of the shell region, as shown by both diffusivity and radial coordination studies. This is consistent with melting temperature reduction due to finite size and surface effects in nanoclusters, but only occurs well above $T_{C}$ in our model.

Regarding our bulk simulations, we obtain an estimated Curie temperature close to $T_{C}=650 \mathrm{~K}$ for bulk systems. The discrepancy with the experimental value is attributed to the exchange coupling $J\left(r_{i j}\right)$. Large differences of the reported values of $J$ as a function of pair separation distance are found in the literature and, therefore, fitting the function $J\left(r_{i j}\right)$ with a different set of $a b$ initio calculations results in different magnetization curves. This statement is clearly verified in Fig. 8 where the bulk Curie temperature is well reproduced if a different set of ab-initio data is used to fit the $J\left(r_{i j}\right)$ function. Our calculations indicate that possible size dependence of $J\left(r_{i j}\right)$ might lead to significant magnetization changes.

The total NP magnetization can be considered to be the result of an ordered core plus a less ordered outer shell. In fact, simple two-component models provide a reasonable fit to our results. Core magnetization resembles bulk magnetization and, as cluster size increases, dominates the overall magnetization. Shell magnetization is significantly lower than bulk magnetization, as expected due to the reduction of the local coordination number.

We propose a functional form for the magnetization as a function of size and temperature, which has only two free parameters and works extremely well for temperatures below $T_{C}$, and for the range of sizes studied, from 2-nm cluster diameter up to bulk conditions.

It is clear that the classical MD-SD simulations presented in this paper still have several limitations, for example, assuming classical continuous spin variables or the fact that exchange, anisotropy, and magnetic moments are the same for surface and core atoms. However, they are expected to contribute to the understanding of magnetism in nanoscale systems, providing quantitative values to compare with experiments for nanocluster magnetization. Among the improvements to be implemented in future studies, it would be interesting to consider the effect of defects in the clusters, such as vacancies, impurities, dislocations and grain boundaries, together with the role of an external magnetic field and dipolar interactions with other nanoclusters.

\section{ACKNOWLEDGMENTS}

We thank Federico Romá for helpful discussionss. E.M.B. thanks support from the SIIP-UNCuyo Grant No. 06/M104. We thank computer run time in the cluster TOKO [88]. The authors thank support from the IPAC-2019 grant from Sistema Nacional de Computación de Alto Desempeño (SNCAD) for run time in the cluster Dirac [89]. Sandia National Laboratories is a multimission laboratory managed and operated by National Technology \& Engineering Solutions of Sandia, LLC, a wholly owned subsidiary of Honeywell International Inc., for the US Department of Energy's National Nuclear Security Administration under Contract No. DE-NA0003525. This paper describes objective technical results and analysis. Any subjective views or opinions that might be expressed in the paper do not necessarily represent the views of the US Department of Energy or the United States Government.
[1] V. Pierron-Bohnes, A. Tamion, F. Tournus, and V. Dupuis, Magnetism of low-dimension alloys, Nanoalloys (Springer, London, 2012), pp. 287-330.

[2] H.-J. Elmers, Ferromagnetic monolayers, Int. J. Mod. Phys. B 9, 3115 (1995).
[3] S. Bader, E. Moog, and P. Grünberg, Magnetic hysteresis of epitaxially-deposited iron in the monolayer range: A Kerr effect experiment in surface magnetism, J. Magn. Magn. Mater. 53, L295 (1986). 
[4] H.-J. Elmers, J. Hauschild, and U. Gradmann, Critical behavior of the uniaxial ferromagnetic monolayer $\mathrm{Fe}(110)$ on $\mathrm{W}(110)$, Phys. Rev. B 54, 15224 (1996).

[5] M. J. Dunlavy and D. Venus, Critical susceptibility exponent measured from Fe/W (110) bilayers, Phys. Rev. B 69, 094411 (2004).

[6] M. Kaur, J. S. McCloy, W. Jiang, Q. Yao, and Y. Qiang, Size dependence of inter-and intracluster interactions in core-shell iron-iron oxide nanoclusters, J. Phys. Chem. C 116, 12875 (2012).

[7] O. Iglesias and A. Labarta, Finite-size and surface effects in maghemite nanoparticles: Monte Carlo simulations, Phys. Rev. B 63, 184416 (2001).

[8] Z. Nedelkoski, D. Kepaptsoglou, L. Lari, T. Wen, R. A. Booth, S. D. Oberdick, P. L. Galindo, Q. M. Ramasse, R. F. Evans, S. Majetich et al., Origin of reduced magnetization and domain formation in small magnetite nanoparticles, Sci. Rep. 7, 45997 (2017).

[9] R. Skomski, Simple Models of Magnetism (Oxford University Press, New York, 2008).

[10] E. C. Stoner and E. Wohlfarth, A mechanism of magnetic hysteresis in heterogeneous alloys, Philos. Trans. R. Soc. London, Ser. A 240, 599 (1948).

[11] B. Zimmermann, G. Bihlmayer, M. Böttcher, M. Bouhassoune, S. Lounis, J. Sinova, S. Heinze, S. Blügel, and B. Dupé, Comparison of first-principles methods to extract magnetic parameters in ultrathin films: Co/Pt(111), Phys. Rev. B 99, 214426 (2019)

[12] F. López-Urías and G. Pastor, Exact diagonalization of hubbard clusters at finite temperatures, Eur. Phys. J. D 52, 159 (2009).

[13] F. Körmann, A. Dick, T. Hickel, and J. Neugebauer, Pressure dependence of the Curie temperature in bcc iron studied by $a b$ initio simulations, Phys. Rev. B 79, 184406 (2009).

[14] V. P. Antropov, M. I. Katsnelson, B. N. Harmon, M. van Schilfgaarde, and D. Kusnezov, Spin dynamics in magnets: Equation of motion and finite temperature effects, Phys. Rev. B 54, 1019 (1996).

[15] A. Vansteenkiste, J. Leliaert, M. Dvornik, M. Helsen, F. GarciaSanchez, and B. Van Waeyenberge, The design and verification of MuMax3, AIP Adv. 4, 107133 (2014).

[16] S. Fu, W. Cui, M. Hu, R. Chang, M. J. Donahue, and V. Lomakin, Finite-difference micromagnetic solvers with the object-oriented micromagnetic framework on graphics processing units, IEEE Trans. Magn. 52, 1 (2016).

[17] G. P. Müller, M. Hoffmann, C. Dißelkamp, D. Schürhoff, S. Mavros, M. Sallermann, N. S. Kiselev, H. Jónsson, and S. Blügel, Spirit: Multifunctional framework for atomistic spin simulations, Phys. Rev. B 99, 224414 (2019).

[18] R. F. Evans, Atomistic spin dynamics, Handbook of Materials Modeling: Applications: Current and Emerging Materials (Springer, Cham, 2020), pp. 427-448.

[19] O. Eriksson, A. Bergman, L. Bergqvist, and J. Hellsvik, Atomistic Spin Dynamics: Foundations and Applications (Oxford university press, Oxford, 2017).

[20] Y. Zhou, J. Tranchida, Y. Ge, J. Murthy, and T. S. Fisher, Atomistic simulation of phonon and magnon thermal transport across the ferromagnetic-paramagnetic transition, Phys. Rev. B 101, 224303 (2020).

[21] X. Wu, Z. Liu, and T. Luo, Magnon and phonon dispersion, lifetime, and thermal conductivity of iron from spin-lattice dynamics simulations, J. Appl. Phys. 123, 085109 (2018).

[22] D. Beaujouan, P. Thibaudeau, and C. Barreteau, Anisotropic magnetic molecular dynamics of cobalt nanowires, Phys. Rev. B 86, 174409 (2012).

[23] R. F. Evans, W. J. Fan, P. Chureemart, T. A. Ostler, M. O. Ellis, and R. W. Chantrell, Atomistic spin model simulations of magnetic nanomaterials, J. Phys.: Condens. Matter 26, 103202 (2014).

[24] M. B. Hahn, Temperature in micromagnetism: Cell size and scaling effects of the stochastic Landau-Lifshitz equation, J. Phys. Commun. 3, 075009 (2019).

[25] S. Westmoreland, R. Evans, G. Hrkac, T. Schrefl, G. Zimanyi, M. Winklhofer, N. Sakuma, M. Yano, A. Kato, T. Shoji et al., Multiscale model approaches to the design of advanced permanent magnets, Scr. Mater. 148, 56 (2018).

[26] D. Suess, C. Vogler, F. Bruckner, P. Heistracher, F. Slanovc, and C. Abert, Spin torque efficiency and analytic error rate estimates of skyrmion racetrack memory, Sci. Rep. 9, 1 (2019).

[27] B. Alkadour, B. W. Southern, J. P. Whitehead, and J. van Lierop, Triangular array of iron oxide nanoparticles: Simulation study of intraparticle and interparticle magnetism, Phys. Rev. B 100, 094416 (2019).

[28] S. Dudarev and P. Derlet, A 'magnetic' interatomic potential for molecular dynamics simulations, J. Phys.: Condens. Matter 17, 7097 (2005).

[29] P. M. Derlet and S. Dudarev, Million-atom molecular dynamics simulations of magnetic iron, Prog. Mater. Sci. 52, 299 (2007).

[30] P.-W. Ma, C. H. Woo, and S. L. Dudarev, Large-scale simulation of the spin-lattice dynamics in ferromagnetic iron, Phys. Rev. B 78, 024434 (2008).

[31] T. Shimada, K. Ouchi, I. Ikeda, Y. Ishii, and T. Kitamura, Magnetic instability criterion for spin-lattice systems, Comput. Mater. Sci. 97, 216 (2015).

[32] P.-W. Ma, S. L. Dudarev, and J. S. Wróbel, Dynamic simulation of structural phase transitions in magnetic iron, Phys. Rev. B 96, 094418 (2017).

[33] P.-W. Ma, S. Dudarev, and C. Woo, Spilady: A parallel CPU and GPU code for spin-lattice magnetic molecular dynamics simulations, Comput. Phys. Commun. 207, 350 (2016).

[34] D. Perera, D. P. Landau, D. M. Nicholson, G. Malcolm Stocks, M. Eisenbach, J. Yin, and G. Brown, Phonon-magnon interactions in body centered cubic iron: A combined molecular and spin dynamics study, J. Appl. Phys. 115, 17D124 (2014).

[35] D. Perera, D. M. Nicholson, M. Eisenbach, G. M. Stocks, and D. P. Landau, Collective dynamics in atomistic models with coupled translational and spin degrees of freedom, Phys. Rev. B 95, 014431 (2017).

[36] J. Hellsvik, D. Thonig, K. Modin, D. Iuşan, A. Bergman, O. Eriksson, L. Bergqvist, and A. Delin, General method for atomistic spin-lattice dynamics with first-principles accuracy, Phys. Rev. B 99, 104302 (2019).

[37] J. Tranchida, S. Plimpton, P. Thibaudeau, and A. P. Thompson, Massively parallel symplectic algorithm for coupled magnetic spin dynamics and molecular dynamics, J. Comput. Phys. 372, 406 (2018).

[38] T. Kaneyoshi, Introduction to Amorphous Magnets (World Scientific Publishing Company, Singapore, 1992). 
[39] K. Yosida, D. C. Mattis, and K. Yosida, Theory of Magnetism: Edition en anglais, Vol. 122 (Springer Science \& Business Media, Berlin, 1996).

[40] M. Pajda, J. Kudrnovsky, I. Turek, V. Drchal, and P. Bruno, $A b$ initio calculations of exchange interactions, spin-wave stiffness constants, and Curie temperatures of $\mathrm{Fe}, \mathrm{Co}$, and Ni, Phys. Rev. B 64, 174402 (2001).

[41] S. Morán, C. Ederer, and M. Fähnle, Ab initio electron theory for magnetism in Fe: Pressure dependence of spin-wave energies, exchange parameters, and Curie temperature, Phys. Rev. B 67, 012407 (2003).

[42] R. F. Sabiryanov and S. S. Jaswal, Magnons and MagnonPhonon Interactions in Iron, Phys. Rev. Lett. 83, 2062 (1999).

[43] W. B. Nurdin and K.-D. Schotte, Dynamical temperature for spin systems, Phys. Rev. E 61, 3579 (2000).

[44] R. Garibay-Alonso, J. Dorantes-Dávila, and G. M. Pastor, Temperature-dependent magnetic properties of ultrathin $\mathrm{Fe}$ films: Interplay between local environment and itinerant ferromagnetism, Phys. Rev. B 73, 224429 (2006).

[45] J. L. García-Palacios and F. J. Lázaro, Langevin-dynamics study of the dynamical properties of small magnetic particles, Phys. Rev. B 58, 14937 (1998).

[46] H. Chamati, N. Papanicolaou, Y. Mishin, and D. Papaconstantopoulos, Embedded-atom potential for $\mathrm{Fe}$ and its application to self-diffusion on Fe $\left(\begin{array}{lll}1 & 0 & 0\end{array}\right)$, Surf. Sci. 600, 1793 (2006).

[47] B. D. Cullity and C. D. Graham, Introduction to Magnetic Materials (John Wiley \& Sons, New Jersey, 2011).

[48] U. Nowak, O. N. Mryasov, R. Wieser, K. Guslienko, and R. W. Chantrell, Spin dynamics of magnetic nanoparticles: Beyond Brown's theory, Phys. Rev. B 72, 172410 (2005).

[49] G. M. Pastor, J. Dorantes-Dávila, S. Pick, and H. Dreyssé, Magnetic Anisotropy of $3 d$ Transition-Metal Clusters, Phys. Rev. Lett. 75, 326 (1995).

[50] M. Ellis and R. Chantrell, Switching times of nanoscale FePt: Finite size effects on the linear reversal mechanism, Appl. Phys. Lett. 106, 162407 (2015).

[51] See Supplemental Material at https://link.aps.org/supplemental/ 10.1103/PhysRevB.xx.xxxxxx for additional figures and movies.

[52] A. Stukowski, Visualization and analysis of atomistic simulation data with OVITO-the open visualization tool, Modell. Simul. Mater. Sci. Eng. 18, 015012 (2009).

[53] D. Mills, Surface effects in magnetic crystals near the ordering temperature, Phys. Rev. B 3, 3887 (1971).

[54] D. S. Bertoldi, E. M. Bringa, and E. N. Miranda, Analytical solution of the mean field Ising model for finite systems, J. Phys.: Condens. Matter 24, 226004 (2012).

[55] D. S. Bertoldi, E. M. Bringa, and E. N. Miranda, Exact solution of the two-level system and the Einstein solid in the microcanonical formalism, Eur. J. Phys. 32, 1485 (2011).

[56] O. Hovorka, S. Devos, Q. Coopman, W. Fan, C. Aas, R. Evans, X. Chen, G. Ju, and R. Chantrell, The Curie temperature distribution of fept granular magnetic recording media, Appl. Phys. Lett. 101, 052406 (2012).

[57] J. Yin, M. Eisenbach, D. M. Nicholson, and A. Rusanu, Effect of lattice vibrations on magnetic phase transition in bcc iron, Phys. Rev. B 86, 214423 (2012).
[58] H. Stanley, Introduction to Phase Transitions and Critical Phenomena the International Series of Monographs on Physics (Oxford University Press Inc., Oxford and New York, 1971).

[59] I. M. L. Billas, J. A. Becker, A. Châtelain, and W. A. de Heer, Magnetic Moments of Iron Clusters with 25 to 700 Atoms and Their Dependence on Temperature, Phys. Rev. Lett. 71, 4067 (1993).

[60] A. V. Ruban and O. E. Peil, Impact of thermal atomic displacements on the Curie temperature of $3 d$ transition metals, Phys. Rev. B 97, 174426 (2018).

[61] L. Bergqvist and A. Bergman, Realistic finite temperature simulations of magnetic systems using quantum statistics, Phys. Rev. Mater. 2, 013802 (2018).

[62] D. Böttcher, A. Ernst, and J. Henk, Temperature-dependent Heisenberg exchange coupling constants from linking electronic-structure calculations and monte carlo simulations, J. Magn. Magn. Mater. 324, 610 (2012).

[63] A. Szilva, M. Costa, A. Bergman, L. Szunyogh, L. Nordström, and O. Eriksson, Interatomic Exchange Interactions for FiniteTemperature Magnetism and Nonequilibrium Spin Dynamics, Phys. Rev. Lett. 111, 127204 (2013).

[64] G. M. Pastor, J. Dorantes-Dávila, and K. H. Bennemann, Size and structural dependence of the magnetic properties of small 3d-transition-metal clusters, Phys. Rev. B 40, 7642 (1989).

[65] G. M. Pastor and J. Dorantes-Dávila, Short-range magnetic order in $\mathrm{Fe}_{N}$ and $\mathrm{Ni}_{N}$ clusters, Phys. Rev. B 52, 13799 (1995).

[66] M. Fisher, in Critical phenomena, edited by M. S. Green (Academic, New York/London), p. 1.

[67] V. Privman, Finite Size Scaling and Numerical Simulation of Statistical Systems (World Scientific Singapore, 1990).

[68] C. Holm and W. Janke, Critical exponents of the classical threedimensional Heisenberg model: A single-cluster Monte Carlo study, Phys. Rev. B 48, 936 (1993).

[69] E. Brézin and J. Zinn-Justin, Finite size effects in phase transitions, Nucl. Phys. B 257, 867 (1985).

[70] J. C. Le Guillou and J. Zinn-Justin, Critical exponents from field theory, Phys. Rev. B 21, 3976 (1980).

[71] J. Le Guillou and J. Zinn-Justin, Accurate critical exponents from the $\varepsilon$-expansion, J. Phys. Lett. 46, 137 (1985).

[72] X. Tao, D. P. Landau, T. C. Schulthess, and G. M. Stocks, Spin Dynamics Simulations of BCC Iron: Spin Dynamics Simulations, Phys. Rev. Lett. 95, 087207 (2005).

[73] E. M. Haines, R. Clauberg, and R. Feder, Short-Range Magnetic Order Near the Curie Temperature Iron from Spin-Resolved Photoemission, Phys. Rev. Lett. 54, 932 (1985).

[74] J. Dorantes-Dávila, G. Pastor, and K. Bennemann, Calculation of short range magnetic order in iron, Solid State Commun. 59, 159 (1986).

[75] B. Martinez and R. Camley, Quantum study of the spin wave spectrum of a superlattice with ferromagnetic interfacial coupling, J. Phys.: Condens. Matter 4, 5001 (1992).

[76] G. Xiao, C. Chien, and M. Natan, Magnetization and its temperature dependence in compositionally modulated amorphous Fe70B30-Ag films, J. Appl. Phys. 61, 4314 (1987).

[77] A. Corciovei, Spin-wave theory of ferromagnetic thin films, Phys. Rev. 130, 2223 (1963).

[78] J.-H. Park, E. Vescovo, H.-J. Kim, C. Kwon, R. Ramesh, and T. Venkatesan, Magnetic Properties at Surface Boundary of a Half-Metallic Ferromagnet $\mathrm{La}_{0.7} \mathrm{Sr}_{0.3} \mathrm{MnO}_{3}$, Phys. Rev. Lett. 81, 1953 (1998). 
[79] D. Zhao, F. Liu, D. L. Huber, and M. G. Lagally, Magnetization on rough ferromagnetic surfaces, Phys. Rev. B 62, 11316 (2000).

[80] L. B. Pártay, On the performance of interatomic potential models of iron: Comparison of the phase diagrams, Comput. Mater. Sci. 149, 153 (2018).

[81] F. Ding, A. Rosén, and K. Bolton, Size dependence of the coalescence and melting of iron clusters: A molecular-dynamics study, Phys. Rev. B 70, 075416 (2004).

[82] P. Ghosh, T. Müller, F. P. Toldin, J. Richter, R. Narayanan, R. Thomale, J. Reuther, and Y. Iqbal, Quantum paramagnetism and helimagnetic orders in the Heisenberg model on the body centered cubic lattice, Phys. Rev. B 100, 014420 (2019).

[83] J.-W. Jang, J. Kwon, and B.-J. Lee, Effect of stress on selfdiffusion in bec Fe: An atomistic simulation study, Scr. Mater. 63, 39 (2010).
[84] R. Gosh, I. M. Syed, Z. Amin, and G. Bhuiyan, A comparative study on temperature dependent diffusion coefficient of liquid Fe, Phys. B: Condens. Matter 426, 127 (2013).

[85] G. M. Bhuiyan, M. Silbert, and M. J. Stott, Structure and thermodynamic properties of liquid transition metals: An embedded-atom-method approach, Phys. Rev. B 53, 636 (1996).

[86] M. D. Kuz'min, Shape of Temperature Dependence of Spontaneous Magnetization of Ferromagnets: Quantitative Analysis, Phys. Rev. Lett. 94, 107204 (2005).

[87] E. A. Velásquez, J. Mazo-Zuluaga, E. Tangarife, and J. MejíaLópez, Structural relaxation and crystalline phase effects on the exchange bias phenomenon in $\mathrm{FeF}_{2} / \mathrm{Fe}$ core/shell nanoparticles, Adv. Mater. Inter. 7, 2000862 (2020).

[88] toko.uncu.edu.ar.

[89] http://dirac.df.uba.ar. 